\title{
A macroscopic link between interhemispheric tract myelination and cortico-cortical interactions during action reprogramming
}

\author{
Alberto Lazari ( $\square$ alberto.lazari@univ.ox.ac.uk) \\ Wellcome Centre for Integrative Neuroimaging, FMRIB, Nuffield Department of Clinical Neurosciences, \\ University of Oxford https://orcid.org/0000-0002-8688-581X
}

Piergiorgio Salvan

University of Oxford

Lennart Verhagen

Donders Institute, Radboud University https://orcid.org/0000-0003-3207-7929

Michiel Cottaar

University of Oxford https://orcid.org/0000-0003-4679-7724

\section{Daniel Papp}

University of Oxford

\section{Olof van der Werf}

Maastricht Brain Imaging Centre (MBIC), Maastricht University, Maastricht, The Netherlands

\section{Bronwyn Gavine}

Wellcome Centre for Integrative Neuroimaging, FMRIB, Nuffield Department of Clinical Neurosciences, University of Oxford James Kolasinski

Cardiff University

\section{Matthew Webster}

University of Oxford

Charlotte Stagg

University of Oxford https://orcid.org/0000-0002-5542-5036

\section{Matthew Rushworth}

University of Oxford https://orcid.org/0000-0002-5578-9884

Heidi Johansen-Berg

University of Oxford

\section{Article}

Keywords: 
Posted Date: December 30th, 2021

DOl: https://doi.org/10.21203/rs.3.rs-333533/v1

License: (c) (i) This work is licensed under a Creative Commons Attribution 4.0 International License. Read Full License

Version of Record: A version of this preprint was published at Nature Communications on July 22nd, 2022. See the published version at https://doi.org/10.1038/s41467-022-31687-5. 


\title{
A macroscopic link between interhemispheric tract myelination and cortico-cortical interactions during action reprogramming
}

\author{
Alberto Lazari ${ }^{1}$, Piergiorgio Salvan ${ }^{1}$, Lennart Verhagen ${ }^{2,3}$, Michiel \\ Cottaar $^{1}$, Daniel Papp ${ }^{1}$, Olof Jens van der Werf ${ }^{4,5}$, Bronwyn \\ Gavine $^{1}$, James Kolasinski ${ }^{6}$, Matthew Webster ${ }^{1}$, Charlotte J \\ Stagg $^{1,7,8}$, Matthew Rushworth ${ }^{2}$, and Heidi Johansen-Berg ${ }^{1}$ \\ ${ }^{1}$ Wellcome Centre for Integrative Neuroimaging, FMRIB, Nuffield Department of Clinical \\ Neurosciences, University of Oxford \\ ${ }^{2}$ Wellcome Centre for Integrative Neuroimaging, Department of Experimental Psychology, \\ University of Oxford \\ ${ }^{3}$ Donders Institute for Brain, Cognition and Behaviour, Radboud University Nijmegen, \\ Nijmegen, Netherlands \\ ${ }^{4}$ Section Brain Stimulation and Cognition, Department of Cognitive Neuroscience, Faculty of \\ Psychology and Neuroscience, Maastricht University, Maastricht, The Netherlands \\ ${ }^{5}$ Maastricht Brain Imaging Centre (MBIC), Maastricht University, Maastricht, The \\ Netherlands \\ ${ }^{6}$ Cardiff University Brain Research Imaging Centre, Maindy Road, Cardiff, CF24 4HQ \\ ${ }^{7}$ Oxford Centre for Human Brain Activity, Wellcome Centre for Integrative Neuroimaging, \\ Department of Psychiatry, University of Oxford \\ ${ }^{8}$ MRC Brain Network Dynamics Unit, University of Oxford, Oxford, OX1 3TH, UK
}

Correspondence: AL: alberto.lazari@ndcn.ox.ac.uk, HJB: heidi.johansen-berg@ndcn.ox.ac.uk

\section{Abstract}

1 Myelination has been increasingly implicated in the function and 2 dysfunction of the adult human brain. Although it is known that axon 3 myelination shapes axon physiology in animal models, it is unclear 4 whether a similar principle applies in the living human brain, and at the 5 level of whole axon bundles in white matter tracts. Here, we 
6 hypothesised that in humans, cortico-cortical interactions between two

7 brain areas may be shaped by the amount of myelin in the white

8 matter tract connecting them. As a test bed for this hypothesis, we

9 use a well-defined interhemispheric premotor-to-motor circuit. We

10 combined TMS-derived physiological measures of cortico-cortical

11 interactions during action reprogramming with multimodal myelin

12 markers (MT, R1, R2* and FA), in a large cohort of healthy subjects.

13 We found that physiological metrics of premotor-to-motor interaction

14 are broadly associated with multiple myelin markers, suggesting

15 interindividual differences in tract myelination may play a role in motor

16 network physiology. Moreover, we also demonstrate that myelination

17 metrics link indirectly to action switching by influencing local primary

18 motor cortex dynamics. These findings suggest that myelination levels

19 in white matter tracts may influence millisecond-level cortico-cortical

20 interactions during tasks. They also unveil a link between the

21 physiology of the motor network and the myelination of tracts

22 connecting its components, and provide a putative mechanism

23 mediating the relationship between brain myelination and human

24 behaviour.

\section{Introduction}

25 Myelination of axonal projections is increasingly being appreciated as a key 26 regulator of brain function. This relationship is widely believed to arise because 27 the properties of an axon's myelination influence many of its physiological 28 properties. Conduction velocity, for instance, increases with myelin thickness 29 and internode length, as demonstrated by observational studies (Brill et al., 30 1977; Rushton, 1951; Waxman, 1980), interventional studies (Caminiti et al., 31 2013; Etxeberria et al., 2016; Schauf and Davis, 1974; Verhoeven et al., 2003) 32 and in silico simulations (Goldman and Albus, 1968; Moore et al., 1978; Smith 33 and Koles, 1970). Moreover, myelination has also been proposed to enable 
34 high-frequency conduction (Saab et al., 2016) and to have a broader role in 35 network physiology (Moore et al., 2019), for example by synchronising neuronal 36 activity (Ford et al., 2015; Seidl et al., 2010) and regulating the timing of 37 action potentials in a circuit-dependent manner (Lang and Rosenbluth, 2003; 38 Pajevic et al., 2014; Salami et al., 2003).

39 While recent advances have allowed the translation of insights on myelination 40 across species (Kaller et al., 2017; Weiskopf et al., 2021), specifically with regards 41 to our understanding of brain plasticity (Sagi et al., 2012; Sampaio-Baptista 42 et al., 2013) and neuroanatomy (Assaf et al., 2020; Glasser et al., 2016; Kirilina 43 et al., 2020; Movahedian Attar et al., 2020), most current evidence directly linking 44 myelination of a circuit to its neurophysiological properties has been derived from 45 animal studies, often focusing on in vitro results. Little is known about how levels 46 of myelination may influence properties of circuits in humans and how this may 47 play out during behaviour. Moreover, many studies have focused on microscopic 48 aspects of myelination, such as individual myelin sheaths, and microscopic aspects 49 of physiology, such as action potentials. However, it is unclear how myelination 50 levels at the macroscopic level of axon bundles influence macroscopic aspects of 51 tract physiology, such as cortico-cortical interactions.

52 In recent years, Magnetic Resonance Imaging (MRI) and Non-Invasive Brain 53 Stimulation (NIBS) have provided new opportunities to tackle questions at 54 macroscopic scales in humans in vivo, which are typically confined to animal or 55 in vitro studies. MRI studies have traditionally aimed to probe properties of 56 white matter through Diffusion-Weighted Imaging (DWI) (Basser et al., 1994; 57 Chenevert et al., 1990; Cleveland et al., 1976; Doran et al., 1990; Le et al., 58 1993; Moseley et al., 1990a,b). DWI, however, provides metrics that are 59 remarkably non-specific to myelination: although it can detect myelin 60 sheath-driven hindered diffusion, it is also sensitive to fiber orientation and 61 other cellular compartments (Zatorre et al., 2012). Recently, several advances 62 have improved our ability to study myelination through MRI (Lazari and Lipp, 63 2021; Mancini et al., 2020). Surging interest in quantifying myelin 64 non-invasively has led to the development of MR sequences sensitive to myelin 
65 through a variety of different biophysical mechanisms: macromolecular tissue 66 content in the myelin lipid bilayer can now be measured by Magnetisation 67 Transfer (MT) (Heath et al., 2018; Sled and Pike, 2001; Tofts et al., 2005; 68 Yarnykh, 2002), local concentrations of diamagnetic myelin and paramagnetic 69 iron-rich oligodendrocytes can be detected through susceptibility-sensitive 70 contrasts (such as R2*) (Weiskopf et al., 2013), and quantification of 71 longitudinal relaxation rates (through R1) makes mapping anatomical variability 72 in myelin content possible (Dubbioso et al., 2021; Lutti et al., 2014). In 73 addition to this new abundance of MR technologies for quantification of 74 myelination, advances in statistics, such as the development of joint inference 75 permutation testing (Winkler et al., 2016) also allow optimal pooling of 76 multimodal data to extract reliable information across these complementary 77 MR signals. While previous work has linked individual cortical myelin-sensitive 78 markers to cortical physiology (Dubbioso et al., 2021), multimodal myelin 79 imaging is a promising tool to draw stronger conclusions about myelination and 80 study myelin's relationship with tract physiology.

81 We hypothesized that MR measures of myelin would relate to physiological 82 measures of cortico-cortical interactions. Although functional MRI (fMRI) has 83 been widely deployed to study cortico-cortical interactions, even advanced 84 computational models of fMRI signals (Friston et al., 2003) cannot detect the 85 directional, causal influence of an area's neuronal activity on the activity of 86 another area. fMRl's low temporal resolution also precludes studying 87 millisecond-level cortico-cortical interactions which are known to underly 88 behaviour (Buch et al., 2010; Lazari et al., 2020a; Neubert et al., 2010). Paired 89 Pulse Transcranial Magnetic Stimulation (ppTMS), by contrast, can be 90 delivered with millisecond precision to allow insights into directional interactions 91 between two brain areas. This high temporal resolution allows ppTMS to detect 92 subtle variation in rapid cortico-cortical interactions, which are not detectable 93 in $\mathrm{fMRI}$ studies. We therefore hypothesized that myelin variability would have 94 fine-grained effects on rapid cortico-cortical interactions, which could be 95 quantified by the use of ppTMS. 
Cortical projections from ventral premotor cortex (PMv) to primary motor 97 cortex (M1) have been extensively characterised both in anatomical tract tracing 98 and physiological studies, across human and non-human primates (for a review, 99 see (Davare et al., 2010)), thus allowing us to formulate detailed hypotheses 100 regarding their function. Behaviourally, these projections allow for inhibition of 101 incorrect motor programmes (Forstmann et al., 2008; Mars et al., 2009, 2007) 102 and are thus engaged during low-level motor tasks such as action reprogramming 103 (Neubert et al., 2010), providing a clear read-out of participant behaviour to 104 relate to their function (Isoda and Hikosaka, 2007). Although there are several 105 premotor areas, two regions on the lateral surface, the dorsal premotor cortex 106 (PMd) and ventral premotor cortex (PMv) stand out because they provide the 107 densest projections to primary motor cortex (M1) (Dum and Strick, 2005). Of 108 these two regions, however, PMv is special. First, its influence over M1 is the best studied; it has been repeatedly demonstrated PMv exerts a strong influence over M1 activity in both non-human and human primates (Cerri et al., 2003;

111 Davare et al., 2008, 2009; Kraskov et al., 2011; Prabhu et al., 2009; Shimazu

112 et al., 2004). This influence can be studied not just in experiments in which $113 \mathrm{PMv}$ is directly stimulated electrically but in transcranial magnetic stimulation 114 (TMS) experiments where the impact of the TMS effect in PMv is especially 115 well characterized; even though the impact of the first pulse in PMv is spatially 116 circumscribed (Romero et al., 2019), it alters the activity in PMv neurons that 117 project to M1 (Cerri et al., 2003; Prabhu et al., 2009; Shimazu et al., 2004). 118 Second, PMv is special not just because it has a strong projection to M1 that is 119 particularly well studied, but, in addition, compared to PMd, PMv receives the 120 stronger projection from prefrontal cortex (Dum and Strick, 2005). This means $121 \mathrm{PMv}$ is well-placed to mediate inhibitory influences exerted over motor control as 122 a result of executive control processes in prefrontal cortical areas. Consistent with 123 such a role, PMv projections to M1 are monosynaptic (Boussaoud et al., 2005; 124 Dum and Strick, 2005; Godschalk et al., 1984; Jenny, 1979) but within M1, many, 125 perhaps the majority, of connections are with inhibitory interneurons as opposed 126 to pyramidal neurons ensuring that PMv is able to exert an inhibitory influence 
127 over M1 (Prabhu et al., 2009; Tokuno and Nambu, 2000). In accordance with 128 such observations, the modulation of M1 by projections from PMv is dependent 129 on behavioural state; PMv exerts a facilitatory influence over M1 during action 130 initiation but an inhibitory influence when no movement is to be made or when 131 an action is to be changed and reprogrammed (Bäumer et al., 2009; Buch et al., 132 2011, 2010; Davare et al., 2008; Neubert et al., 2010). Moreover, these inhibitory 133 influences have been linked to specific white matter tracts connecting PMv and 134 M1 (Neubert et al., 2010), thus providing a clear anatomical location not just 135 in cortex but in underlying white matter at which to investigate PMv-to-M1 136 modulation. While many anatomical tracing studies focus only on ipsilateral 137 connections within a hemisphere, PMv has many transcallosal connections with 138 heterotopic areas in the other hemisphere (Dancause et al., 2007; Lanz et al., 139 2017) and consistent with this anatomical observation it is established that PMv 140 exerts similar facilitatory and inhibitory influences over the activity of M1 both 141 ipsilaterally and contralaterally (Buch et al., 2011, 2010; Johnen et al., 2015;

142 Neubert et al., 2010; Sel et al., 2021). Therefore, the PMv-to-M1 circuit can

143 also be studied interhemispherically, thus making it possible to probe mechanisms

144 that involve fibers passing through the corpus callosum, and that may be unique 145 to interhemispheric circuits (Assaf et al., 2020; Bachtiar et al., 2018; Calford 146 and Tweedale, 1990; Fling et al., 2013; Krupnik et al., 2021; van der Knaap and 147 van der Ham, 2011). Taken together, these features of PMv-to-M1 projections 148 (the presence of both clear behavioural and clear physiological read-outs, the link 149 to a defined interhemispheric white matter tract) make them an ideal test bed for 150 hypotheses about how myelin shapes circuit function and behaviour in humans.

151 Here, we aimed to test whether inhibitory interactions between PMv and 152 M1 (as measured through ppTMS) may be shaped by the amount of myelin in 153 the white matter tract connecting them (as measured by multimodal myelin 154 markers). We also aimed to probe the relationship between white matter 155 myelination, cortico-cortical inhibition, and behavioural output in an action 156 reprogramming task. Because previous experiments suggest that white matter 157 myelination rarely relates directly to behavioural performance (Lazari et al., 
158 2020b), we hypothesized that during action reprogramming, PMv-to-M1 159 inhibition may link white matter myelination to behavioural performance. As 160 PMv-to-M1 projections are known to modulate the balance between excitation 161 and inhibition within M1 (Prabhu et al., 2009; Tokuno and Nambu, 2000), we 162 also hypothesized that PMv-to-M1 inhibition and local inhibition within M1 163 may both contribute to behavioural output during action reprogramming. 


\section{Methods}

\section{Participants}

16464 healthy participants ( 36 female; mean age 24.69 years) underwent a single 165 session of MRI and a separate TMS session which included task-based paired 166 pulse TMS. TMS and MRI sessions were matched for time-of-day and were 167 separated by a maximum of one week. All participants were self-assessed 168 right-handed and their handedness was further confirmed through the 169 Edinburgh Handedness Inventory (Oldfield et al., 1971), (mean EHI score: 170 88.65). All participants were screened for TMS and MRI safety, received 171 monetary compensation for their participation, and gave their informed consent 172 to participate in this study. All study procedures were reviewed and approved by 173 the local ethics committee at the University of Oxford (Central University 174 Research Ethics Committee (CUREC)), and followed the Declaration of 175 Helsinki.

\section{Measuring myelination: acquisition of Magnetic Resonance Imaging (MRI)}

176 Magnetic Resonance Imaging (MRI) data were collected with a 3.0-T Prisma

177 Magnetom Siemens scanner, software version VE11C (Siemens Medical

178 Systems, Erlangen, Germany). T1-weighted structural imaging (T1w),

179 Multi-Parameter Mapping (MPM) and Diffusion-Weighted Imaging (DWI) 180 sequences were collected.

181 The $\mathrm{T} 1 \mathrm{w}$ sequence $(\mathrm{TR}=1900 \mathrm{~ms}$, $\mathrm{TE}=3.96 \mathrm{~ms}$, voxel size $=1 \mathrm{~mm}$ 182 isotropic, GRAPPA $=2$ ) had a large Field of View $\left(F O V=256 \mathrm{~mm}^{3}\right)$ to allow

183 for the nose and intertragic notches of the ears to be included in the image to 184 facilitate later neuronavigation of the TMS coil to the target position.

185 Diffusion-weighted Echo-planar imaging (EPI) scans ( $\mathrm{TR}=3070 \mathrm{~ms}$, TE $=$ $18685.00 \mathrm{~ms}$, FOV $=204 \mathrm{~mm}^{3}$, voxel size $=1.5 \mathrm{~mm}$ isotropic, multiband factor of 187 4) were collected for two b-values (500 and $2000 \mathrm{~s} / \mathrm{mm}^{2}$ ), over 281 directions. 
188 An additional 23 volumes were acquired at $b=0,15$ in anterior-posterior (AP) 189 phase-encoding direction and 8 in the posterior-anterior (PA) phase-encoding 190 direction.

191 The MPM protocol (Weiskopf et al., 2013) included three multi-echo 3D 192 FLASH (fast low-angle shot) scans with varying acquisition parameters, one RF 193 transmit field map (B1+map) and one static magnetic (B0) field map scan, 194 for a total acquisition time of roughly 22 minutes. To correct for inter-scan 195 motion, position-specific receive coil sensitivity field maps, matched in FOV to 196 the MPM scans, were calculated and corrected for (Papp et al., 2016). The 197 three types of FLASH scans were designed to be predominantly T1-, PD-, or 198 MT-weighted by changing the flip angle and the presence of a pre-pulse: 8 199 echoes were predominantly Proton Density-weighted ( $T R=25 \mathrm{~ms}$; flip angle $200=6$ degrees; TE $=2.3-18.4 \mathrm{~ms}), 8$ echoes were predominantly T1-weighted $201 \quad(\mathrm{TR}=25 \mathrm{~ms}$; flip angle $=21$ degrees; $\mathrm{TE}=2.3-18.4 \mathrm{~ms})$ and 6 echoes were 202 predominantly Magnetisation Transfer-weighted (MTw, TR $=25 \mathrm{~ms}$; flip angle $203=21$ degrees; TE $=2.3-13.8 \mathrm{~ms})$. For MTw scans, excitation was preceded by 204 off-resonance Gaussian MT pulse of 4 ms duration, nominal flip angle, $2 \mathrm{kHz}$ 205 frequency offset from water resonance. All FLASH scans had $1 \mathrm{~mm}$ isotropic 206 resolution and field of view (FOV) of $256 \times 224 \times 176 \mathrm{~mm}^{3}$. The B1 map was 207 acquired through an EPI-based sequence featuring spin and stimulated echoes 208 (SE and STE) with 11 nominal flip angles, FOV of $192 \times 192 \times 256 \mathrm{~mm}^{3}$ and TR of $209500 \mathrm{~ms}$. The TE was $37.06 \mathrm{~ms}$, and the mixing time was $33.8 \mathrm{~ms}$. The B0 map 210 was acquired to correct the B1+ map for distortions due to off-resonance effects. 211 The B0 map sequence had a TR of $1020.0 \mathrm{~ms}$, first TE of $10 \mathrm{~ms}$, second TE of $21212.46 \mathrm{~ms}$, field of view (FOV) of $192 \times 192 \times 256 \mathrm{~mm}^{3}$ and read-out bandwidth of $213260 \mathrm{~Hz} /$ pixel.

214 MRI scan pre-processing, analysis and statistical comparisons were performed 215 using FMRIB Software Library (FSL, v6.0), except for the MPM quantitative map 216 estimation step which was carried out using the hMRI toolbox implemented in 217 Matlab-based SPM, as described in (Tabelow et al., 2019). All T1w images were 218 preprocessed through a standard FreeSurfer-based pipeline (Fischl et al., 2004; 
219 Glasser et al., 2013) to correct for bias field and achieve ACPC alignment (for 220 use in Neuronavigation).

221 An automated custom pipeline based on existing FSL tools was also developed 222 for our diffusion sequence. The topup tool was run on average images of AP b0 223 volumes and PA b0 volumes. The resulting susceptibility-induced off-resonance 224 field was used as an input for the eddy tool (Andersson and Sotiropoulos, 2016), 225 which was run with options optimised for multiband diffusion data to correct for 226 eddy currents and subject movement. To generate Fractional Anistropy (FA) 227 maps, a diffusion tensor model was fit to each voxel through DTIFIT, optimised 228 for multi-shell data processing with options such as -kurt.

229 Magnetisation Transfer saturation (MT), R1 and R2* quantitative maps 230 were estimated through the hMRI toolbox (Tabelow et al., 2019). In order to 231 register MPM volumes to FA volumes, we used the following steps. 232 Boundary-Based Registration was used to calculate a DWI-to-T1w registration 233 using AP b0 images (with high tissue boundary contrast). A customised 234 pipeline was used to ACPC align the MPM maps and register them to T1w 235 space. At this stage, one participant was excluded as the MPM scan was 236 heavily corrupted due to movement artefacts; one participant was excluded due 237 to lower quality signal in the MPM scan, which resulted in poor registrations 238 with other modalities. Once registration matrices for MPM-T1w and DWI-T1w 239 were calculated, they were inverted, concatenated and applied as needed to 240 bring MPM volumes into DWI space with minimal interpolation.

\section{Measuring myelination: joint inference across myelin-sensitive MRI modalities}

241 To bring all volumes into a common space, native FA volumes were skeletonised 242 with Tract-Based Spatial Statistics (TBSS), and the skeletonisation transforms 243 were subsequently applied to MPM-to-DWI registered volumes. Group-level 244 analyses were then conducted in skeleton space for all data, and using 245 rank-based inverse-normal transformations on the regressors. To uncover 
246 common trends across modalities (i.e. trends compatible with an effect of 247 myelin), voxelwise joint inference was performed through Permutation Analysis 248 of Linear Models, which implements a voxelwise Fisher test with the following 249 equation (as described in (Winkler et al., 2016)):

$$
-2 \sum_{K=1}^{K} \ln \left(p_{k}\right)
$$

250 with $p_{k}$ being each modality's $p$-value and $K$ being the total number of 251 modalities being combined (full code available here: 252 https://git.fmrib.ox.ac.uk/alazari/macroscopic-link).

253 As a second step, to extract a single myelin metric from all myelin markers 254 for use in the comparisons between correlations and in the mediation analyses, 255 we used dimensionality reduction through Principal Component Analysis 256 (PCA). The follow-up analyses focused on voxels identified as significant 257 through joint inference; within these voxels, fslmeants was used to extract the 258 mean value for each modality. A principal component analysis was then used in 259 order to reduce the dimensionality of the normalised data, an approach already 260 previously described for white matter data (Chamberland et al., 2019). As the 261 first principal component captured $69.22 \%$ of total variance, the subject-level 262 scores for the first principal component were then used as the predictor variable 263 in the mediation analysis (full code available here:

264 https://git.fmrib.ox.ac.uk/alazari/macroscopic-link).

\section{Measuring action reprogramming}

265 The Action Reprogramming task was implemented based on a task originally 266 developed for monkeys (Isoda and Hikosaka, 2007, 2008), and then adapted for 267 use in human TMS studies (Mars et al., 2009; Neubert et al., 2010). The task 268 aimed to probe action execution and action reprogramming. Cues consisted of 269 a central square (either red or green) with two 'flanker' squares (one red and 270 one green). Participants were instructed to press the button on the side where 271 the flanker colour matched the colour of the central square. The flankers kept 
272 switching sides at random, whereas the central square was always the same colour 273 for 3-7 consecutive trials at the time. The central square was white when first 274 appearing on the screen, and then turned green/red 450-600ms after the flankers 275 were presented. Thus the $450-600 \mathrm{~ms}$ interval allowed participants to generate a 276 motor plan prior to motor execution towards either the left or right flanker given 277 the expectation that they had about what the central cue colour change would 278 be. This way, participants simply had to execute a pre-prepared movement when 279 the central cue colour stayed the same ('stay trials'), but they had to inhibit the 280 movement and carry out a different one in the trials where the central cue colour 281 had switched ('switch trials'). All stimulus presentation timings mirrored ones 282 used in previous literature (Neubert et al., 2010), including the inter-trial interval 283 (1000 ms) and intervals between flanker and cue presentation (450-600 ms). 284 Each participant underwent a session of 112 switch trials and corresponding stay 285 trials (for a total of 678 trials), resulting in 28 TMS stimulations per condition in 286 each participant (Goldsworthy et al., 2016). Each session included 3 automatic 287 breaks in between to ensure participants had a chance to rest if they wished to 288 do so.

289 Participants were told to be as fast and accurate as they could. They 290 received detailed task instructions in paper format at the beginning of each 291 session; in addition, the instructions were reiterated in a computer-based 292 fashion at the beginning of the task (full code available here: 293 https://git.fmrib.ox.ac.uk/alazari/macroscopic-link). Before the task, they 294 undertook roughly 100 trials to make sure first that they understood the rules 295 of the task, that they had habituated to the task, and that they could tolerate 296 TMS pulses while performing the task. Participants were pressing the two 297 buttons with index fingers from their two hands, and were instructed to keep 298 their hands relaxed between trials until the cue appeared; they were 299 occasionally reminded to keep their hands relaxed if the experimenter observed 300 noticeable muscle contractions between trials.

301 Our primary behavioural measure was the ratio between reaction time between 302 switch trials and stay trials. We shall refer to this as 'switch RT cost'. Switch 
303 RT cost is calculated as follows:

$$
\text { Switch RT Cost }=\frac{\mu_{\text {Switch Trial RTs }}}{\mu_{\text {Stay Trial RTs }}}
$$

304 where $\mu$ is the mean of the Reaction Times (RTs) being considered. Switch RT 305 cost always has a positive value; the higher the value, the higher the switch RT 306 cost.

\section{Measuring cortico-cortical inhibition: Paired pulse TMS (ppTMS)}

307 Detailed methods for Transcranial Magnetic Stimulation (TMS) and 308 neuronavigation set-ups are described in Supplementary Methods. In brief, two 309 DuoMAG MP-Dual TMS monophasic stimulators (DeyMed DuoMag, Rogue 310 Resolutions Ltd.) were used to deliver pulses via two figure-eight coils, one over 311 left M1 and one over right PMv. All stimulation was delivered using continuous 312 tracking of coil location with respect to subject neuroanatomy (i.e. 313 neuronavigation) using a Polaris camera and the Brainsight (Rogue Resolutions, 314 Inc.) software. Electromyography (EMG) was recorded from the participant's 315 right hand in a tendon-belly montage, in order to record TMS-induced 316 Motor-Evoked Potentials (MEPs) from the hand's First Dorsal Interosseus 317 muscle (see Supplementary Methods). TMS was delivered during an Action 318 Reprogramming task (see above).

319 Cortico-cortical interactions were measured by paired pulse TMS (ppTMS), 320 as follows. On half of all stimulation trials, participants were stimulated over 321 left M1 only; on the other half of trials, the same stimulation was preceded 322 by a conditioning right PMv pulse $6 \mathrm{~ms}$ earlier. Although MEPs reflect the 323 connectivity between M1 and the periphery, our focus in the current report is 324 not on the MEPs per se but on their modulation by the induction of activity 325 within PMv which a number of studies in both non-human and human primates 326 demonstrate reflect the interactions between PMv and M1 (Cerri et al., 2003; 327 Davare et al., 2008, 2009; Kraskov et al., 2011; Prabhu et al., 2009; Romero et al., 
328 2019; Shimazu et al., 2004). As both the M1 single pulse condition and the paired 329 pulse PMv-M1 condition elicited the same level of peripheral stimulation, the 330 overall paradigm allows us to control for corticospinal and peripheral influences 331 (Civardi et al., 2001).

332 We decided on a 6 ms inter-pulse interval (IPI) based on previous work 333 (Neubert et al., 2010) and after exclusion of other potential IPIs. We excluded 334 higher IPIs, as IPIs of 9-18 ms have been shown to engage poly-synaptic 335 subcortical pathways (Neubert et al., 2010). We also excluded lower IPIs, as 336 they reflect variability in inhibitory intracortical circuits (which are engaged at 337 1-4 ms IPI) and would likely not engage transcallosal fibers sufficiently (Kujirai 338 et al., 1993; Stagg et al., 2011). Together with evidence from other ppTMS 339 studies on this circuit (Davare et al., 2008, 2009), we concluded that focussing 340 on a single IPI of $6 \mathrm{~ms}$ was the ideal strategy to isolate the contribution of 341 transcallosal myelinated fibers to the ppTMS effect.

342 The pulse over M1 was always delivered $175 \mathrm{~ms}$ after cue onset - based on 343 previous experiments (Neubert et al., 2010) - and set at $1 \mathrm{mV}$ intensity (see 344 Supplementary Methods); the pulse over PMv was always delivered at 110\% 345 of resting Motor Threshold (rMT, see Supplementary Methods). Pulses were 346 randomly assigned in an equal fashion to stay vs switch trials (when pre-prepared 347 actions were either executed or inhibited) and to right-hand vs left-hand trials.

348 EMG analysis focused on TMS-induced MEPs, and more specifically on the 349 peak-to-peak amplitude of MEPs. MEP recording, preprocessing and analysis 350 procedures were identical for all experiments. MEPs were identified based on 351 recorded TMS timings, pre-processed in MATLAB (Version R2018b, Mathworks, 352 MA, USA) and visualised for quality control purposes. Absent or unusually small $353 \operatorname{MEPs}(<0.2 \mathrm{mV})$ or unusually high MEPs $(>9 \mathrm{mV})$ were excluded from further 354 analyses. The average rejection rate for unusually small MEPs was $1.25 \%$, and $3550.84 \%$ for unusually high MEPs. Trials with incorrect, premature $(<150 \mathrm{~ms})$ or 356 slow ( $>800 \mathrm{~ms}$ ) responses were also excluded from further analyses. Trials with 357 significant 'precontraction' (i.e. muscle contraction above $0.4 \mathrm{mV}$ in the $100 \mathrm{~ms}$ 358 before the MEP and the test pulse) were also excluded. Once all exclusion criteria 
359 were applied, Grubb's outlier detection procedure was carried out on the data. 6 360 participants had fewer than 9 MEPs per condition which could be included, and 361 were thus excluded from further analyses.

362 After preprocessing, MEP ratios (paired pulse TMS/single pulse TMS) were 363 obtained from the median normalised MEP in switch trials. We shall refer to 364 this as 'switch PP/SP ratio'. Similar to previous studies (Neubert et al., 2010), 365 calculation of this metric for switch trials did not involve any data from stay 366 trials, in order to minimise the influence from excitatory processes happening 367 during action execution (Buch et al., 2010; Neubert et al., 2010). Switch PP/SP 368 ratio was used as our primary measures of cortico-cortical inhibition, and was 369 calculated as follows:

$$
\text { Switch PP/SP ratio }=\frac{M d_{\text {Switch Trial Paired Pulse MEPs }}}{M d_{\text {Switch Trial Single Pulse MEPs }}}
$$

370 Where Md is the median of the total MEPs being considered. Switch PP/SP ratio 371 always has a positive value; the lower the value, the higher the interhemispheric 372 inhibition.

\section{Measuring task-dependent inhibition of Primary Motor Cortex (M1)}

373 To obtain a more fine-grained picture of dynamics in $M 1$, we estimated the extent 374 of its task-dependent inhibition. This was done by stimulating the motor hotspot 375 at $1 \mathrm{mV}$ with single pulse TMS; in half the TMS trials this was done during stay 376 trials, and in the other half this was done during switch trials. The pulse delivered 377 to M1 was always delivered $175 \mathrm{~ms}$ after cue onset and pulses were randomly 378 assigned in an equal fashion to stay vs switch trials. We find that MEPs driven 379 by $\mathrm{M} 1$ stimulation decrease significantly during switch (action reprogramming) 380 trials compared to stay (action execution) trials (Wilcoxon matched-pairs rank 381 test: median of differences: -0.2849 , 95\% Confidence Interval of median of 382 differences: -0.3955 to $-0.1563, \mathrm{p}<0.001$ ).

383 The ratio between median MEP on switch and stay trials was calculated for 
384 each subject, and used as our primary measure of task-dependent inhibition of 385 M1. We shall refer to this as 'switch M1 inhibition'. Switch M1 inhibition was 386 calculated as follows:

$$
\text { Switch } M 1 \text { inhibition }=\frac{M d_{\text {Switch Trial Single Pulse MEPs }}}{M d_{\text {Stay }} \text { Trial Single Pulse MEPs }}
$$

387 Where Md is the median of the total MEPs being considered.

\section{Tractography of stimulated white matter fibers}

388 White matter bundles connecting the stimulated cortical sites were estimated 389 through Probtrackx (Behrens et al., 2007). Regions of interest (ROI) in the 390 cortex were based on the neuronavigation-derived sites for each participant (see 391 Supplementary Methods). This ensured that in each subject, the region of 392 interest used for tractography corresponded to the site that was stimulated via 393 TMS. As the motor hotspot does not always overlap with the postcentral gyral 394 fold, and a larger coil was used for M1 compared to PMv, the motor hotspot $395 \mathrm{ROI}$ was enlarged to a $3 \mathrm{~cm}$ radius to improve the output tract quality. 396 Tractography was run in native DWI space, with outputs in Montreal 397 Neurological Institute (MNI) space to enable pooling of results across all 398 subjects. Individual-level maps of streamline densities were then thresholded at $3991 \%$ of the number of valid streamlines and overlaid.

\section{Statistical comparison between correlations and Mediation analysis}

400 Differences between correlations were tested in the R package psych, using 401 Fisher's z-transformed correlation coefficients. Based on previous results (Lazari 402 et al., 2020b; Neubert et al., 2010), we hypothesized that the correlation 403 between myelin and switch PP/SP ratio would be greater than correlations

404 between myelin and other metrics. Given the directionality of this hypothesis, a 405 one-tailed test was used. As all correlations tested originated from the same 
406 sample, a paired test was used to account for the dependency between 407 correlations being compared.

408 Mediation analyses were run in PROCESS (to derive 95\% Confidence Intervals 409 (Hayes, 2013)) and in BRAVO (to derive p-values (Wager et al., 2008), which 410 cannot be derived in PROCESS for our model). In both toolboxes, we used a 411 significant indirect ( $\mathrm{X}-\mathrm{M})$ pathway as the key requirement to test mediation, and 412 applied bootstrapping with 10000 repetitions (Hayes, 2013; Zhao et al., 2010). 


\section{Results}

\section{Multimodal myelin markers, interhemispheric cortico-cortical inhibition, and action reprogramming}

413 We hypothesized that greater myelination of a long-range projection (measurable 414 with multimodal MRI markers of myelin) is associated with stronger physiological 415 interactions between cortical target areas (measurable with ppTMS), and that 416 this, in turn, is associated with better behavioural performance. MRI allows 417 collection of many quantitative markers that are known to be related to myelin 418 (Figure 1A). Taken independently, each marker is sensitive to other features of 419 the tissue (e.g. R2* is also sensitive to iron), so a joint inference test was used to 420 find common patterns across all MRI markers. Since myelin is the only common 421 biological feature all sequences are sensitive to, we reasoned that any concordant, 422 common trend in relationships with all these markers must signify strong evidence 423 for a myelin-driven effect.

424 ppTMS (Figure 1B) was used to probe levels of directional inhibition from 425 right ventral premotor cortex (PMv) to left primary motor cortex (M1) during 426 the action reprogramming task (Figure 1C, D). On half of all stimulation trials, 427 participants were stimulated over M1 only, to determine their average Motor428 Evoked Potential during single pulse stimulation. On the other half of trials, 429 M1 stimulation was preceded by PMv stimulation, with the aim of quantifying 430 the effect that activating PMv neurons would have on M1-driven Motor-Evoked 431 Potential. By taking a ratio between Motor-Evoked Potentials in single pulse (M1 432 only) stimulation and paired stimulation (PMv and M1) during switch trials, we 433 obtained the switch PP/SP ratio, a metric of the interaction between the two 434 areas (Figure 1B). 
Cortico-cortical inhibition (paired pulse TMS)



Joint inference across myelin markers (Fisher test)

$$
-2 \sum_{K=1}^{K} \ln \left(p_{k}\right)
$$


C Action Reprogramming Task



D
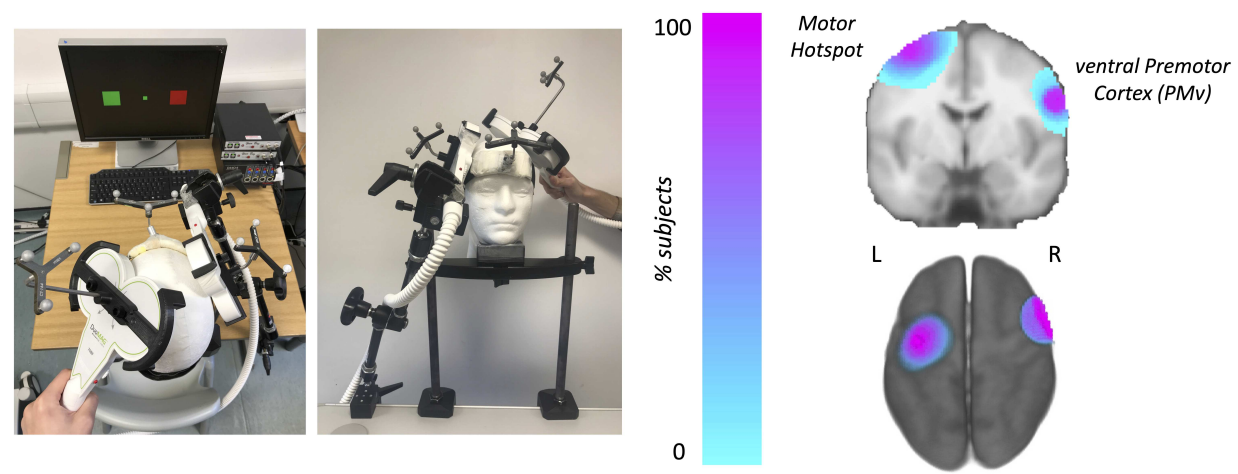
Figure 1: (Previous page.) Non-invasive measurement of myelination and of cortico-cortical interactions during behaviour. A. Multiple myelinsensitive MRI modalities were collected and jointly analysed through multimodal joint inference. B. Motor-Evoked Potentials were collected from the same participants during an action reprogramming task. Stimulation of premotor cortex during the task allowed measurement of the modulatory effects of premotor cortex on Motor-Evoked Potentials driven by M1 stimulation. More specifically, in a Single Pulse condition (SP, left) a test pulse (red) over left motor cortex elicits a Motor-Evoked Potential in the right hand; in a Paired Pulse condition (PP, right) the test pulse (red) is preceded by a conditioning pulse (blue) over right ventral premotor cortex. The ratio of hand Motor-Evoked Potential amplitude between the Single Pulse and Paired Pulse conditions during switch trials (switch PP/SP ratio) is our measure of cortico-cortical interaction. C. Schematic of the action reprogramming task. The ratio between reaction times in stay trials and switch trials (switch RT cost) is our primary behavioural measure. D. Transcranial Magnetic Stimulation set-up used to measure corticocortical interactions. Precise neuroanatomical targeting is achieved through continuous neuronavigation during stimulation, and confirmed with analysis of subject-specific target locations of stimulation (right).

\section{Physiological measures of interhemispheric cortico-cortical inhibition relate to myelination of the underlying long-range circuit}

435 As hypothesised, we found that people exhibiting the most inhibition from PMv 436 to $M 1$, as measured by a smaller switch PP/SP ratio, also have higher levels of 437 myelin markers in white matter (Figure $2 \mathrm{~A}$, peak $\mathrm{p}_{\text {FisherFWE }}=0.016$ ), with 438 correlations especially prominent in MT, R1 and R2* $(r=-0.3839, r=-0.4120$ 439 and $r=-0.4304$ respectively, Figure $2 B$ ). Within our whole-brain analysis, the 440 areas where this correlation is significant are spatially asymmetric (Figure 2A). 
441 In the left hemisphere, they cluster posteriorly, under the average location of 442 the M1 coil; in the right hemisphere, they cluster slightly more anteriorly, under 443 the average location of the PMv coil. Finally, the significant clusters in both 444 hemispheres are connected by a cluster crossing the midline in the corpus 445 callosum. This hints that myelination of the tract being stimulated may be 446 uniquely important for PMv's inhibition of M1 during action reprogramming.

447 To probe the specificity of the relationship between myelination and 448 cortico-cortical interactions, we then ask whether this observed relationship is 449 unique to the physiological interactions between cortical areas, or whether 450 myelination also relates to local physiological inhibition in $\mathrm{M} 1$, or to behavioural 451 performance during action reprogramming (Figure S1). To test this, we used 452 joint inference again and found that behavioural switch RT cost during the 453 action reprogramming task does not significantly relate to myelin markers (peak $\left.454 \mathrm{p}_{\text {FisherFWE }}=0.058\right)$. Switch M1 inhibition also does not significantly relate to 455 myelin markers (peak $p_{\text {FisherFWE }}=0.192$ ). Finally, myelin markers are 456 significantly less correlated to behavioural switch RT cost and switch M1 457 inhibition, than they are to switch PP/SP ratio $(p=0.0128$ and $p=0.0007$, 458 respectively). In summary, the correlations with myelin markers that we 459 identified are specifically related to physiological measures of PMv-to-M1 460 inhibition - and they are not as strong for physiological measures of M1 461 inhibition or for behavioural measures that may reflect the integrated output of 462 a broader set of brain regions rather than just PMv and M1. 


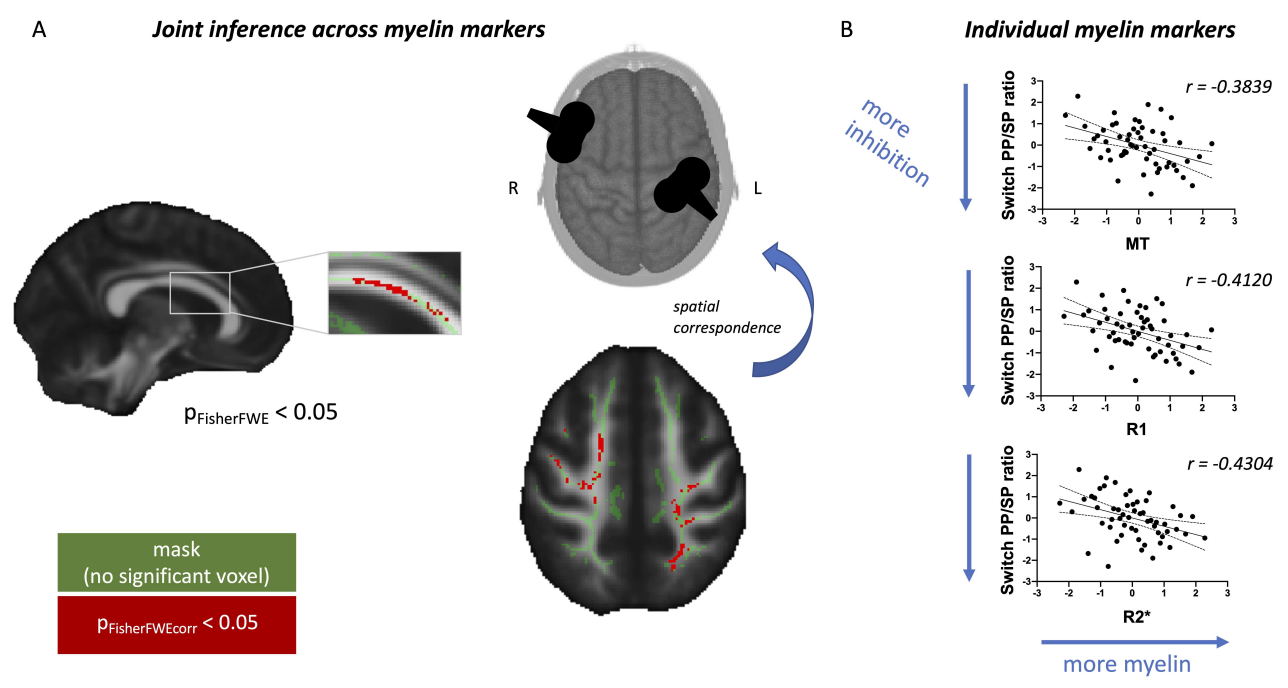

Figure 2: Multimodal joint inference reveals concordant relationships between inter-hemispheric cortico-cortical inhibition and myelin markers across modalities. A: Result of joint inference across myelin markers. Clusters of significant voxels are shown in red, overlaid on the white matter skeleton (green). Significance threshold for joint inference Fisher test is set at 0.05 after correcting for family-wise error correction. B. Correlations between switch PP/SP ratio (paired pulse/single pulse ratio from ppTMS) and individual myelin markers. Each data point is a single participant; scatterplots and Spearman correlation effect sizes are presented for post-hoc visualisation of the correlations, rather than for statistical inference.

\section{Interhemispheric cortico-cortical inhibition mediates the} link between myelin markers and action reprogramming behaviour

463 Cortico-cortical interactions are constrained by structural features of 464 cortico-cortical connections (Grandjean et al., 2017; Hermundstad et al., 2013). 465 Moreover, cortico-cortical connectivity shapes the dynamics of individual brain 
466 areas (Bachtiar et al., 2018; Lindenberg et al., 2013), and in turn individual 467 brain areas perform computations that directly regulate behavioural output. 468 This is especially clear in the case of M1, whose computations directly 469 determine movement (Churchland and Shenoy, 2007; Elsayed et al., 2016) and 470 are input-driven (Sauerbrei et al., 2020). Moreover, the inputs to M1 are also 471 well-described, with excitatory premotor projections synapsing onto M1 472 interneurons (Buch et al., 2010; Davare et al., 2010; Tokuno and Nambu, 473 2000). Therefore, we take advantage of the well-established circuit properties 474 of the motor network to hypothesize that cortico-cortical inhibition from right 475 PMv to left M1 would relate to local cortical inhibition in M1, and that in turn 476 M1 inhibition would relate to motor behaviour.

477 In support of this hypothesis (Figure S2), we find that inhibition from PMv 478 to $\mathrm{M} 1$, as measured by the switch $\mathrm{PP} / \mathrm{SP}$ ratio, correlates with switch M1 479 inhibition $(r=-0.5245, \mathrm{p}<0.0001)$, and that switch $\mathrm{M} 1$ inhibition correlates 480 with behavioural output, as captured by switch RT cost $(r=-0.3096$, $481 \mathrm{p}=0.0202$ ), but we find no significant relationship between ppTMS inhibition 482 and switch RT cost $(r=0.1925, p=0.1551)$. Moreover, as mentioned previously, 483 myelination correlates specifically with inhibition from $P M v$ to $M 1$, but not with 484 switch M1 inhibition, nor with behavioural switch RT cost ( $p_{\text {FisherFWE }}>0.05$, 485 Figure S1).

486 Given our strong, directional, a priori hypotheses on how myelination, 487 cortico-cortical inhibition and action reprogramming behaviour would relate to 488 each other, we formalise these relationships in a single statistical model, by 489 means of a 2-mediator mediation analysis (Figure 3). In cross-sectional designs, 490 mediation analysis does not replace causal or pseudo-causal approaches, but is 491 a powerful tool to test statistical inter-dependence between variables in a 492 hypothesis-driven way, as we do here. In accordance with our hypothesis, we 493 find that inhibition from PMv to M1, as measured by the switch PP/SP ratio 494 (mediator 1) and switch M1 inhibition (mediator 2) sequentially mediate the 495 link between myelination and action reprogramming behaviour $(\mathrm{p}=0.0427$, $49695 \%$ boostrapped $\mathrm{Cl}:-.29$ to -0.02 , indirect link explaining $61.8 \%$ of the total 
497 effect). Finally, we find this mediation result to be specific to switch RT cost, 498 as the same mediation does not explain variability in stay trial Reaction Times $499(\mathrm{p}=0.4488$, Figure S4). 

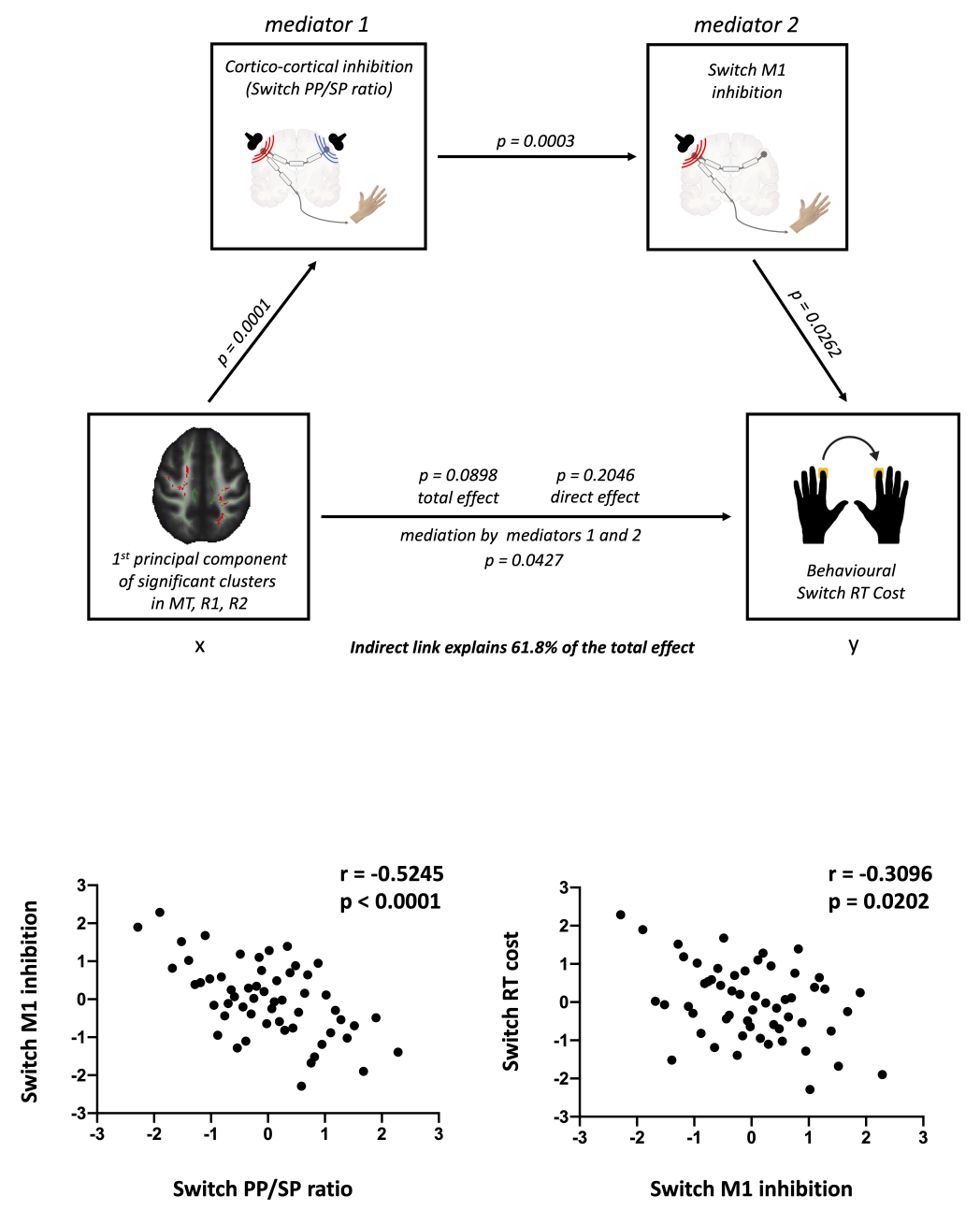

Figure 3: Motor network physiology links myelination to switching behaviour. A: Mediation analysis reveals an indirect link between the myelinrelated measure of inter-hemispheric cortico-cortical inhibition (Box X) and switch RT cost, a metric of performance during the action reprogramming task (Box $\mathrm{Y}$ ). The $95 \%$ boostrapped $\mathrm{Cl}$ for the indirect effect of sequential mediation of $\mathrm{M} 1$ and $\mathrm{M} 2$ is -.29 to -0.02 , which overall explained $61.8 \%$ of the total effect. B: Inhibition from PMv to M1, as measured by $\mathrm{ppTMS}$, correlates with switch M1 inhibition $(r=-0.5245, p<0.0001)$. C: Switch M1 inhibition correlates with action reprogramming behaviour, as measured by switch RT cost $(r=-0.3096$, $\mathrm{p}=0.0202)$. 


\section{Tractography of stimulated white matter tracts based on individual stimulation sites}

500 We then investigated the anatomical location of sites where myelin markers

501 correlated with physiological measures of cortico-cortical interactions. By using

502 information from individual participants' cortical stimulation sites, we

503 performed tractography to reconstruct estimates of the stimulated white matter

504 fibers (Figure 4, Supplementary Video 1). We find that in all individuals, the

505 stimulated white matter fibers run interhemispherically through the body of the

506 corpus callosum. Consistent with our hypotheses, the clusters where

507 myelination markers correlate with stimulation-based physiological readouts are

508 located in anatomical white matter areas that were consistently stimulated

509 across all subjects. 
Real-time neuronavigation
to capture individual stimulation sites


Individual level tractography of stimulated white matter fibers

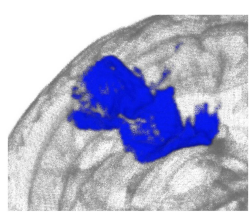

$\Rightarrow$


Group-level tractography Multimodal cluster $p_{\text {corr }}<0.05$

Figure 4: Tractography of stimulated white matter fibers. A. We estimate individual-level and group-level maps of white matter fibers stimulated in our paradigm. B. Left: The group level map of tract overlap across subjects is shown in blue, overlaid on a coronal section. Locations for sagittal sections are indicated in green. Right: Sagittal sections showing anatomical overlap between stimulated fibers (blue) and the cluster where myelin metrics correlate with stimulation-based metrics of cortico-cortical interactions (red). 


\section{Physiological measures of interhemispheric cortico-cortical inhibition do not correlate with demographic factors and features of M1 physiology}

510 To probe whether the switch PP/SP ratio relates to non-specific individual 511 differences across subjects, we tested correlations between switch PP/SP ratio, 512 demographic factors, and features of primary motor cortex physiology (Figure 513 S4). We found that switch PP/SP ratio does not correlate with resting Motor 514 Threshold ( $r M T, r=-0.2, p=0.87$ ), active Motor Threshold (aMT, $r=0.10$, $515 \mathrm{p}=0.46)$ or $1 \mathrm{mV}(\mathrm{r}=-0.3, \mathrm{p}=0.82)$ measures. We also find that age and gender 516 are not correlated with any other physiological metric, and in particular not 517 with switch PP/SP ratio (age: $r=0.01, p=0.94$; gender: $r=0.004, p=0.98$ ). 


\section{Discussion}

518 It has been repeatedly suggested that variability of white matter in the general

519 population holds explanatory value concerning individual differences in brain

520 physiology (Boorman et al., 2007; Neubert et al., 2010), cognition (Matejko

521 and Ansari, 2015), and behaviour (Boekel et al., 2015; Kanai and Rees, 2011).

522 However, the features of white matter that are driving this meaningful

523 variability are less well understood (Lazari et al., 2020b). In recent years, white

524 matter myelination has come to prominence as an underappreciated regulator of

525 brain function, with studies demonstrating new roles such as trophic support of

526 axons (Fünfschilling et al., 2012; Nave, 2010) and also plastic potential

527 (Sampaio-Baptista et al., 2013). Moreover, several strains of evidence have

528 highlighted that myelination influences cognition and behaviour in ageing and in

529 a range of pathologies (Cabibel et al., 2020; Forbes et al., 2020; Hill et al.,

530 2018; Lakhani et al., 2017; Ruckh et al., 2012; Shen et al., 2008; Weil et al.,

531 2016; Zonouzi et al., 2015). Therefore, it would make sense for inter-individual

532 variability in myelin to also play an important role in cognition and behaviour in

533 health (Dubbioso et al., 2021), and potentially to underpin the previously

534 reported relevance of white matter variability to wide-ranging behaviours. This

535 study provides a direct test of that prediction, demonstrating that variability in

536 white matter myelination of healthy adults holds meaningful explanatory

537 information about physiological and behavioural processes, and opens the door

538 to further studies exploring inter-individual variability of myelination in the

539 general population.

540 In the current study, we focused on the relationship between myelination

541 of a well-defined white matter circuit (measured with multimodal MRI), the

542 downstream physiological activity within that circuit (measured with ppTMS),

543 and the resulting behaviour (measured with an Action Reprogramming Task).

544 We find a link between metrics of physiological interactions between two brain

545 areas and metrics of white matter myelination in the tract connecting the two

546 areas. This relationship proves highly specific. It is common across multiple

547 myelin markers, and a whole-brain voxelwise analysis reveals it to be localised 
548 to areas of the pathways stimulated by ppTMS. Taken together, these results 549 argue for a specific link between the physiology of PMv to M1 projections, their 550 myelination, and the action reprogramming behaviour they support.

551 Although the study highlights a robust relationship between the amount of 552 myelin in a tract and its physiological properties, it is difficult to interpret which 553 morphological aspects of a tract may be driving this effect. Levels of myelination 554 in a voxel are influenced by a variety of factors, including myelin thickness, number 555 of oligodendrocytes, length of Nodes of Ranvier (NoR), and axon packaging 556 (Walhovd et al., 2014). As a simplified example, a voxel with high MT could 557 reflect a high number of myelinated axons, or thicker myelin sheaths on each axon 558 (Kaller et al., 2017; Walhovd et al., 2014; Zatorre et al., 2012). Recent work has 559 also highlighted the contributions that NoRs may play in dynamically regulating 560 functional axonal properties (Arancibia-Carcamo et al., 2017; Dutta et al., 2018;

561 Ford et al., 2015; Lazari et al., 2018), which our results are compatible with. To 562 further the previous example, two voxels with the exact same number of myelin 563 sheets and oligodendrocytes may have different MT and R2* values if one of the 564 voxels has shorter NoRs, therefore allowing more myelin to cluster within the same 565 3D volume (Andersson et al., 2020; Cottaar et al., 2020; Walhovd et al., 2014). 566 In summary, our study is compatible with multiple morphological interpretations 567 within white matter, but cannot distinguish between them. Future studies will 568 be able to build upon our findings to tackle these biologically-driven questions by 569 reverse-translation to rodent studies, where tools such as immunohistochemistry 570 and electron microscopy would be able to answer more detailed cellular questions. 571 A key limitation of the study is that it employs non-invasive, MR-based 572 metrics of myelination to quantify myelin. Drawing one-to-one relationships 573 between biophysical signals detected from MR and the underlying biology is 574 notoriously problematic (Walhovd et al., 2014; Zatorre et al., 2012), and we 575 took several steps to maximise the biological interpretability of our findings. 576 Rather than focusing on an individual myelin marker, we collected a suite of 577 four different myelin markers, each with a different profile of biophysical 578 sensitivity (Lazari and Lipp, 2021). We also used a joint inference framework 
579 when carrying out statistical analyses, so that we would only consider effects 580 that are common across modalities. This minimises the risk that the link we 581 discovered here is driven by other biological features of white matter: MT is 582 also sensitive to oedema (Cook et al., 2004), and R2* is also sensitive to 583 vasculature (Weisskoff and Kiihne, 1992), but the only known signal that they 584 both pick up is from tissue myelination. Although it comes with limitations, 585 using non-invasive markers of myelination allows the gathering of insights and 586 testing of hypotheses in humans that otherwise would be restricted to 587 preclinical studies in animal models. In particular, taking this approach has 588 allowed us to identify a new link between human behaviour and myelination 589 that would have been inaccessible through invasive histology.

590 A foundational assumption of many in vitro studies of axonal properties is 591 that the same principles that apply to relationships between an axon's 592 morphology and its physiological properties play out in similar ways at a larger 593 scale in axon bundles within white matter tracts. The current results provide

594 further confirmation of this assumption. Previous studies have found that 595 increased myelination boosts conduction velocity and information transmission 596 between individual neurons. Here, by probing cortico-cortical circuits with 597 macroscopic imaging and stimulation methods, we show that a similar 598 relationship exists for white matter tracts and cortex-to-cortex connectivity, 599 whereby increased myelination of a tract also influences physiological 600 interactions of one cortical area with another.

601 We studied brain-behaviour relationships in the context of a particular 602 behavioral task - namely action reprogramming. Controlling what actions to 603 execute, often termed 'cognitive control' (Neubert et al., 2010), is a key 604 function of the brain, and is likely an agglomeration of a variety of parallel and 605 diverse mechanisms (Chambers et al., 2006; Forstmann et al., 2008; 606 MacDonald et al., 2000; Mars et al., 2007; Ridderinkhof et al., 2004). We 607 examined a specific component of 'cognitive control', i.e. action reprogramming 608 (Isoda and Hikosaka, 2007). Here, we show that at least some components of 609 cognitive control can be explained in terms of physiological interactions, which 
610 are in turn driven by myelination of the underlying circuit. This constitutes a

611 fundamental step forward in our understanding of the mechanisms underpinning

612 cognitive control, and how we may be able to modulate them.

613 Our findings also suggest that interindividual variability in tract myelination, 614 tract physiology, and behaviour, are closely linked to each other. Subjects with 615 higher levels of myelination exhibited stronger inhibition and lower switch RT 616 costs during action reprogramming. Conversely, subjects with lower levels of 617 myelination exhibited reduced inhibition and higher switch RT costs during 618 action reprogramming. One potential interpretation of the finding could be that 619 the chosen inter-pulse interval of $6 \mathrm{~ms}$ is optimal in the participants with more 620 myelin, and that participants with less myelin have delayed inhibition. This is in 621 agreement with our data, as some subjects showed facilitation from right PMv 622 to left M1, suggesting that their physiological inhibition is either lowered or 623 delayed during action reprogramming. While additional imaging modalities 624 (such as Magnetic Resonance Spectroscopy) would be needed to disentangle 625 what precise features of cortical or white matter physiology lead to dampened 626 inhibition in these subjects, our result suggest that participants displaying 627 absent inhibition during action reprogramming also have higher switch RT cost, 628 and that this phenomenon does therefore contribute to behavioural output.

629 These results illustrate a mechanistic link binding myelination of long-range 630 circuits to their physiology and to action reprogramming. They highlight, for 631 the first time, that myelin's effects on axon conductance are also relevant at 632 the larger spatial scales of tracts and translatable to the study of the live 633 human brain. Taken together, this represents a first step towards translating 634 studies on cellular-level properties of myelin to human-relevant knowledge. This 635 is crucial for developing clinically-relevant insights into cortical circuits and to 636 design therapeutic treatments aimed at modulating myelination. 


\section{Supplementary Results}



Supplementary Figure 1: Relationships between white matter myelination, and physiological and behavioural measures. We use joint inference and find that ppTMS-based measures of cortico-cortical interactions (i.e. switch PP/SP ratio) significantly correlate with myelin markers $\left(p_{\text {FisherFWE }}<0.05\right)$, whereas behavioural switch RT cost and switch M1 inhibition do not significantly correlate with myelin markers ( $\left.p_{\text {FisherFWE }}>0.05\right)$. 
A

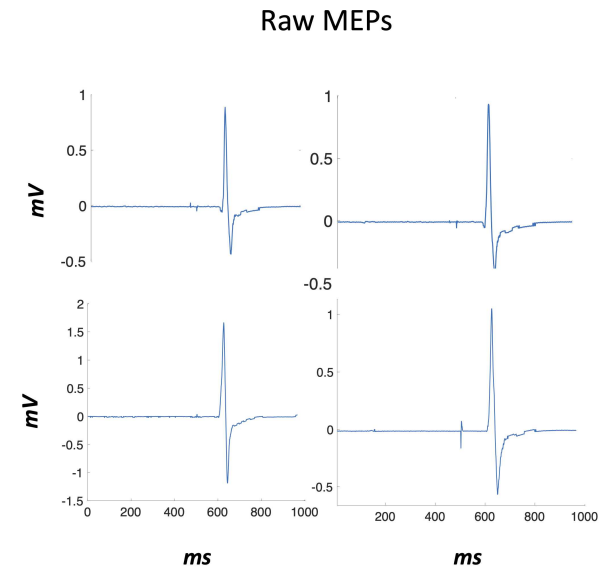

B



C



Supplementary Figure 2: Raw Motor-Evoked Potentials (MEPs) across conditions. A. Raw EMG traces for randomly selected MEPs from the dataset. B. Raw MEP amplitudes used to derive the switch PP/SP ratio; 29 out of 56 subjects had smaller MEPs in PP compared to SP trials during switch trials. C. Raw MEP amplitudes used to derive switch M1 inhibiton. 


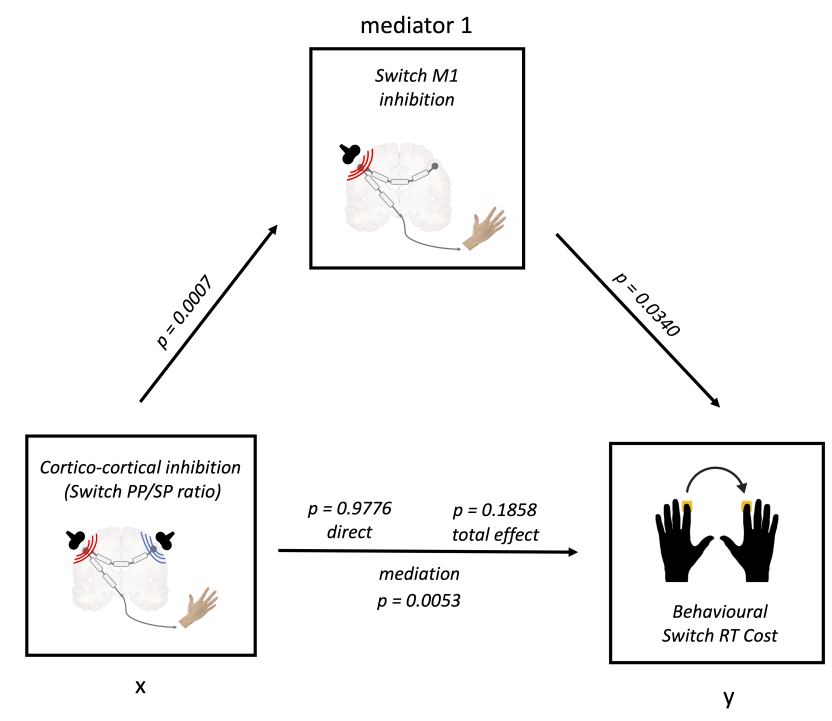

Supplementary Figure 3: 1-mediator mediation analysis. To complement the full 2-mediator analysis, we show that 1-mediator analysis (with no neuroimaging data) yields similar results. In this analysis, we use switch M1 inhibition as a mediator of the relationship between cortico-cortical interactions (ppTMS metric) and behaviour output (switch RT cost). 


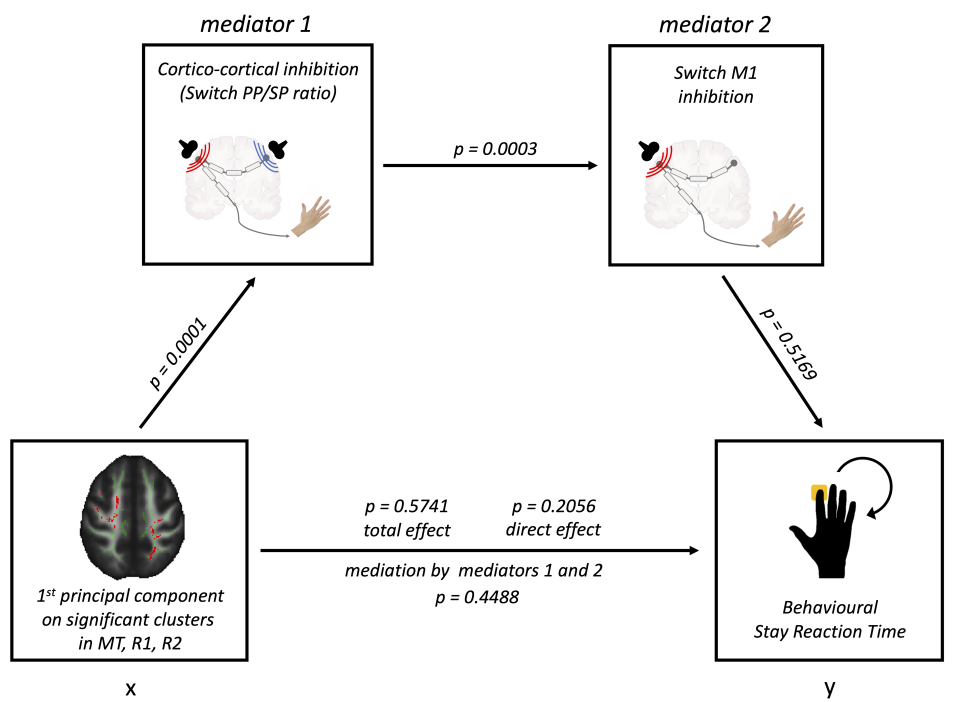

Supplementary Figure 4: Mediation analysis for stay trial Reaction Times.

To test the behavioural specificity of the mediation analysis result, we repeated the analysis with stay trial Reaction Times as our outcome measure, and found no significant mediation effect $(p=0.4488)$. 


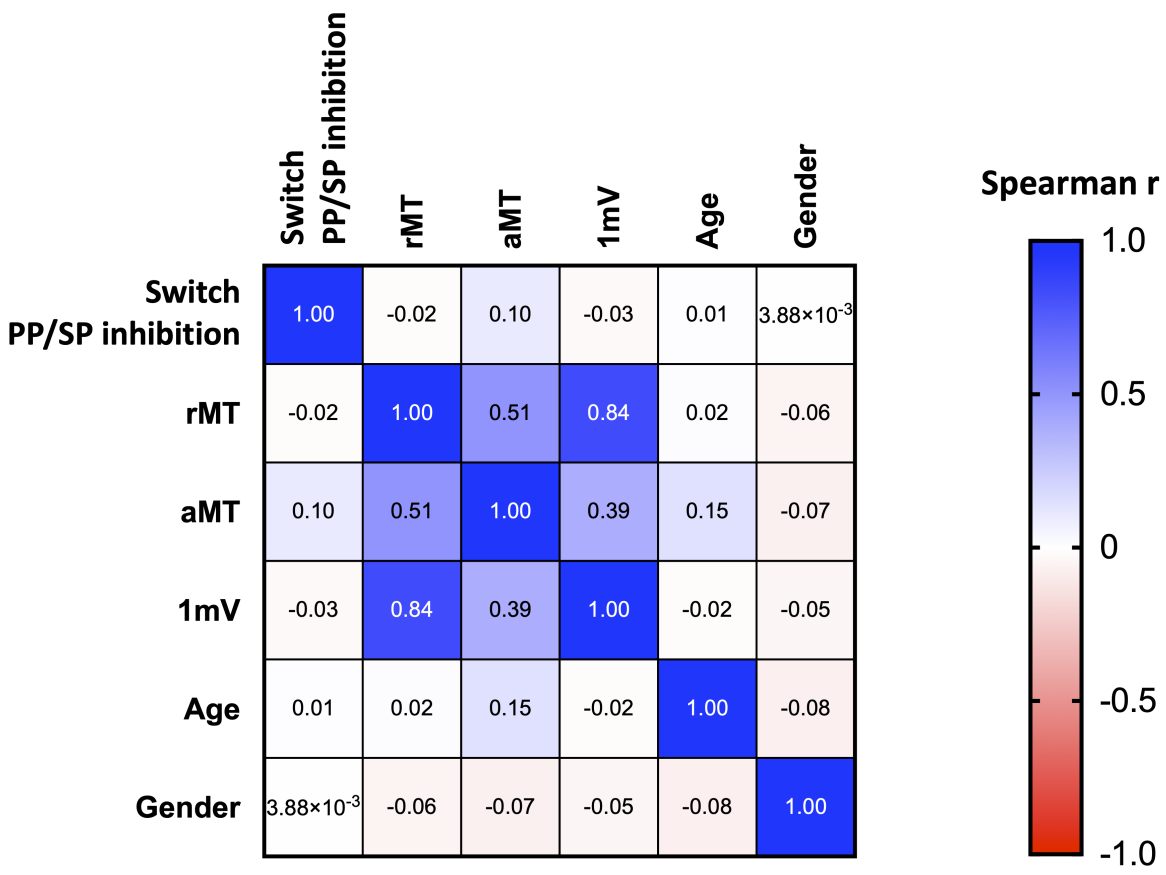

Supplementary Figure 5: Correlations between cortico-cortical inhibition (switch PP/SP ratio), demographic factors and features of M1 physiology. We report Spearman $r$ correlation values for correlations of all possible pairs between: switch PP/SP ratio, resting Motor Threshold (rMT), active Motor Threshold (aMT), $1 \mathrm{mV}$, age and gender. We find no significant correlation for the switch $\mathrm{PP} / \mathrm{SP}$ ratio metric across any tests. 


\section{Supplementary Methods}

\section{Transcranial Magnetic Stimulation (TMS) set-up}

637 Two DuoMAG MP-Dual TMS monophasic stimulators (DeyMed DuoMag, Rogue

638 Resolutions Ltd.) were used to deliver paired pulses via two figure-eight coils, 639 one $70 \mathrm{~mm}$-diameter coil over left M1 and one $50 \mathrm{~mm}$-diameter coil over right 640 PMv.

641 The $70 \mathrm{~mm} / \mathrm{M} 1$ coil was held by the main experimenter throughout the 642 experiment. This coil was kept tangential to the skull and at roughly an $45^{\circ}$ 643 angle to the scalp's midline, resulting in a Posterior-to-Anterior (PA) current 644 direction induced in the cortex. The $50 \mathrm{~mm}$ PMv coil was positioned in place 645 through a clamp sustained by a Manfrotto Variable Friction Arm (Wex Photo 646 Video, Calumet Photographic Limited) which was clamped to the experiment

647 table. The position of the coil was determined by registration of its location to 648 the participant's T1w image, and targeted coordinates $x=58, y=15, z=30$ 649 in MNI (Montreal Neurological Institute) space, based on (Neubert et al., 650 2010). The angle of the PMv coil was $0^{\circ}$ relative to midline.

651 Participants sat on a chair and were asked to position their head on a chin rest 652 in order to minimize head movement. Before the start of any TMS stimulation, 653 participants were asked to keep their feet relaxed and flat on the ground. The 654 participants were free to move their body between task blocks, but they were 655 asked to move the hand with the electrodes as little as possible both during the 656 task and between task blocks. All participants wore earplugs to reduce the effects 657 of TMS-related noise.

\section{Using Neuronavigation to track stimulation sites}

658 All stimulation was delivered using continuous tracking of coil location with 659 respect to subject neuroanatomy (i.e. neuronavigation). This was achieved 660 through a Polaris camera and the Brainsight (Rogue Resolutions, Inc.) 661 software. The participant was tracked via a headband with reflective spheres 
662 attached to it; the coils were tracked with coil trackers that were re-calibrated 663 at the beginning of each testing day. In addition, extensive hard-ware checks 664 were performed before each session, including that the coils worked on a 665 peripheral muscle, that cable connections were correctly in place, and 666 importantly, a PicoScope6 (Pico Technology) was used to check the timing of 667 the two pulses to ensure they were correct and identical between task and rest 668 blocks at sub-millisecond precision.

669 Online neuronavigation was used in all subjects to ensure the coil was 670 targeting the cortical area of choice throughout the task. Moreover, a sample 671 of the coil location was collected for each participant during the session, and 672 analysed offline. An automated Brainsight tool was used to find the closest 673 brain voxel to the sampled stimulation site. The coordinates for this voxel were 674 then transformed in standard space to allow overlaying of stimulation sites from 675 different participants. At this stage, a total of 47 stimulation locations were 676 included, as two participants' stimulation locations failed to save due to 677 software fault, three participants's stimulation locations were not sampled due 678 to time limits, and four participant's stimulation locations could not be 679 automatically determined with Brainsight. Because the magnetic field may 680 reach $30 \%$ of its peak level throughout a region with a diameter of $4 \mathrm{~cm}$ 681 (Siebner et al., 2009), spheres of $4 \mathrm{~cm}$ diameter were created around the 682 sample stimulation location to provide a conservative estimate of the spatial 683 specificity achieved by TMS. These spheres were then overlaid upon each other 684 (Figure 1). All stimulation sites were within $1 \mathrm{~cm}$ of target location, as 685 described in previous publications (Buch et al., 2011; Johnen et al., 2015).

\section{Electromyography (EMG)}

686 Electromyography (EMG) was recorded from the participant's right hand in a 687 tendon-belly montage, to record from the First Dorsal Interosseus muscle. After 688 scrubbing the three electrode sites with alcohol wipes, 25-mm electrodes 689 (Kendall Neonatal ECG Electrodes Puppydog) were applied, with the ground 690 electrode placed on the hand's carpus. In order, the EMG signal output was 
691 processed through a D440 amplifier (Digitimer), a Humbug Noise Eliminator, $69250 \mathrm{~Hz}$ (Digitimer) to notch-filter the data, and a CED micro1401 Mk.II A/D 693 converter (Cambridge Electronic Design) to digitise the signal and relay it to a 694 PC running Spike2 (Cambridge Electronic Design). Sampling rate was $5000 \mathrm{~Hz}$, 695 and bandpass filters were set between 10 and $1000 \mathrm{~Hz}$.

\section{Motor hotspot and parameter determination}

696 The M1 coil intensity was increased and slowly moved around over the left side 697 of the scalp until an M1 motor hotspot could be identified. Several criteria 698 were applied to confirm the correct coil location had been reached: reliability 699 of the MEPs, smoothness of the MEP shape, selectivity of finger movement 700 during MEPs, and degradation of MEP response as the coil moved away from

701 the identified spot. A subset of tested participants failed to meet all of our motor

702 hotspot criteria, and thus did not complete the protocol and were excluded from 703 any TMS-related analysis. The location of the motor hotspot was recorded 704 through neuronavigation during the first session. After the first session, the 705 recorded location was used to quickly re-confirm the motor hotspot; this also 706 ensured the same motor hotspot was stimulated across sessions in multi-day 707 protocols.

708 After determining the location of the motor hotspot, three parameters were 709 determined for each participant: the intensities for $1 \mathrm{mV}, \mathrm{rMT}$ and aMT. $1 \mathrm{mV}$ 710 was determined as the intensity giving reliable and stable $1 \mathrm{mV}$ MEPs at rest over 711 approximately 10 pulses; stability of the MEPs, rather than a precise mean value 712 of $1 \mathrm{mV}$ across 10 pulses, was used as the key parameter in determining $1 \mathrm{mV}$ 713 intensity. rMT was determined as the intensity at which 5 out of 10 pulses gave 714 no MEP response greater than $0.05 \mathrm{mV}$.

715 After $1 \mathrm{mV}$ and $\mathrm{rMT}$ determination, a brief, standardised protocol was used 716 to determine aMT (Stagg et al., 2011). A separate screen was moved towards 717 the participant so that they could observe their own FDI EMG trace in real 718 time from the chinrest. After the participants verbally confirmed they could see 719 the screen, they were asked to squeeze the thumb against the index finger as 
720 hard as they could twice to determine their maximum contraction. A sliding 721 line on the screen was then set to $20 \%$ of maximum muscle contraction, and 722 participants were asked to try and keep their contraction levels around that line. 723 If this level of contraction caused fatigue or was too close to the noise level, then 724 maximum contraction was calculated once again. This FDI-contraction set-up 725 allowed measurement of the aMT, which we defined in this experiment as the 726 intensity at which 5 out of 10 TMS pulses produced an MEP that was time727 locked to the TMS pulse, and was followed by a cortical silent period. Presence 728 of time-locked MEPs, rather than presence of cortical silent period, was used as 729 the key parameter in determining aMT. 


\section{Acknowledgements}

We are grateful to Alex S. Bates, Antoine Cherix, Ilona Lipp and Laia Serratosa Capdevila for their input on previous versions of the manuscript. We would like to thank Martina Callaghan from University College London for the MPM sequence. We would also like to thank Juliet Semple, Nicola Aikin and Sebastian Rieger for their technical support and help with scanning participants; and Matthew Webster for wide-ranging help from IT to statistical issues. We acknowledge the IT-related support provided by David Flitney and Duncan Mortimer throughout the project.

\section{Funding}

This work was supported by a PhD Studentship awarded to AL from the Wellcome Trust $(109062 / Z / 15 / Z)$ and by a Principal Research Fellowship from the Wellcome Trust to HJB (110027/Z/15/Z). CJS holds a Sir Henry Dale Fellowship, funded by the Wellcome Trust and the Royal Society $(102584 / Z / 13 / Z)$. JK is supported by a Sir Henry Wellcome Postdoctoral Fellowship (204696/Z/16/Z). The project was supported by the NIHR Oxford Health Biomedical Research Centre. TMS work was supported by the University of Oxford John Fell Fund and the University of Oxford Wellcome Trust Institutional Strategic Support Fund (to CJS). The Wellcome Centre for Integrative Neuroimaging is supported by core funding from the Wellcome Trust $(203139 /$ Z/16/Z). 


\section{References}

Andersson, J. L. and Sotiropoulos, S. N. (2016). An integrated approach to correction for off-resonance effects and subject movement in diffusion $\mathrm{mr}$ imaging. Neuroimage, 125:1063-1078.

Andersson, M., Kjer, H. M., Rafael-Patino, J., Pacureanu, A., Pakkenberg, B., Thiran, J.-P., Ptito, M., Bech, M., Dahl, A. B., Dahl, V. A., et al. (2020). Axon morphology is modulated by the local environment and impacts the noninvasive investigation of its structure-function relationship. Proceedings of the National Academy of Sciences, 117(52):33649-33659.

Arancibia-Carcamo, I. L., Ford, M. C., Cossell, L., Ishida, K., Tohyama, K., and Attwell, D. (2017). Node of ranvier length as a potential regulator of myelinated axon conduction speed. Elife, 6:e23329.

Assaf, Y., Bouznach, A., Zomet, O., Marom, A., and Yovel, Y. (2020). Conservation of brain connectivity and wiring across the mammalian class. Nature neuroscience, 23(7):805-808.

Bachtiar, V., Johnstone, A., Berrington, A., Lemke, C., Johansen-Berg, H., Emir, U., and Stagg, C. J. (2018). Modulating regional motor cortical excitability with noninvasive brain stimulation results in neurochemical changes in bilateral motor cortices. Journal of Neuroscience, 38(33):7327-7336.

Basser, P. J., Mattiello, J., and LeBihan, D. (1994). Mr diffusion tensor spectroscopy and imaging. Biophysical journal, 66(1):259-267.

Bäumer, T., Schippling, S., Kroeger, J., Zittel, S., Koch, G., Thomalla, G., Rothwell, J., Siebner, H., Orth, M., and Münchau, A. (2009). Inhibitory and facilitatory connectivity from ventral premotor to primary motor cortex in healthy humans at rest-a bifocal tms study. Clinical Neurophysiology, 120(9):1724-1731. 
Behrens, T. E., Berg, H. J., Jbabdi, S., Rushworth, M. F., and Woolrich, M. W. (2007). Probabilistic diffusion tractography with multiple fibre orientations: What can we gain? Neuroimage, 34(1):144-155.

Boekel, W., Wagenmakers, E.-J., Belay, L., Verhagen, J., Brown, S., and Forstmann, B. U. (2015). A purely confirmatory replication study of structural brain-behavior correlations. Cortex, 66:115-133.

Boorman, E. D., O'Shea, J., Sebastian, C., Rushworth, M. F., and JohansenBerg, H. (2007). Individual differences in white-matter microstructure reflect variation in functional connectivity during choice. Current Biology, 17(16):1426-1431.

Boussaoud, D., Tanné-Gariépy, J., Wannier, T., and Rouiller, E. M. (2005). Callosal connections of dorsal versus ventral premotor areas in the macaque monkey: a multiple retrograde tracing study. BMC neuroscience, 6(1):67.

Brill, M., Waxman, S., Moore, J., and Joyner, R. (1977). Conduction velocity and spike configuration in myelinated fibres: computed dependence on internode distance. Journal of Neurology, Neurosurgery \& Psychiatry, 40(8):769-774.

Buch, E. R., Johnen, V. M., Nelissen, N., O'Shea, J., and Rushworth, M. F. (2011). Noninvasive associative plasticity induction in a corticocortical pathway of the human brain. Journal of Neuroscience, 31(48):17669-17679.

Buch, E. R., Mars, R. B., Boorman, E. D., and Rushworth, M. F. (2010). A network centered on ventral premotor cortex exerts both facilitatory and inhibitory control over primary motor cortex during action reprogramming. Journal of Neuroscience, 30(4):1395-1401.

Cabibel, V., Héraud, N., Perrey, S., Oliver, N., Alexandre, F., and Varray, A. (2020). Is bilateral corticospinal connectivity impaired in patients with chronic obstructive pulmonary disease? The Journal of Physiology, 598(20):45914602. 
Calford, M. B. and Tweedale, R. (1990). Interhemispheric transfer of plasticity in the cerebral cortex. Science, 249(4970):805-807.

Caminiti, R., Carducci, F., Piervincenzi, C., Battaglia-Mayer, A., Confalone, G., Visco-Comandini, F., Pantano, P., and Innocenti, G. M. (2013). Diameter, length, speed, and conduction delay of callosal axons in macaque monkeys and humans: comparing data from histology and magnetic resonance imaging diffusion tractography. Journal of Neuroscience, 33(36):14501-14511.

Cerri, G., Shimazu, H., Maier, M., and Lemon, R. (2003). Facilitation from ventral premotor cortex of primary motor cortex outputs to macaque hand muscles. Journal of neurophysiology, 90(2):832-842.

Chamberland, M., Raven, E. P., Genc, S., Duffy, K., Descoteaux, M., Parker, G. D., Tax, C. M., and Jones, D. K. (2019). Dimensionality reduction of diffusion mri measures for improved tractometry of the human brain. Neurolmage, 200:89-100.

Chambers, C. D., Bellgrove, M. A., Stokes, M. G., Henderson, T. R., Garavan, H., Robertson, I. H., Morris, A. P., and Mattingley, J. B. (2006). Executive ?brake failure? following deactivation of human frontal lobe. Journal of cognitive neuroscience, 18(3):444-455.

Chenevert, T. L., Brunberg, J. A., and Pipe, J. G. (1990). Anisotropic diffusion in human white matter: demonstration with $\mathrm{mr}$ techniques in vivo. Radiology, 177(2):401-405.

Churchland, M. M. and Shenoy, K. V. (2007). Temporal complexity and heterogeneity of single-neuron activity in premotor and motor cortex. Journal of neurophysiology, 97(6):4235-4257.

Civardi, C., Cantello, R., Asselman, P., and Rothwell, J. C. (2001). Transcranial magnetic stimulation can be used to test connections to primary motor areas from frontal and medial cortex in humans. Neuroimage, 14(6):1444-1453. 
Cleveland, G., Chang, D., Hazlewood, C., and Rorschach, H. (1976). Nuclear magnetic resonance measurement of skeletal muscle: anisotrophy of the diffusion coefficient of the intracellular water. Biophysical journal, 16(9):10431053.

Cook, L. L., Foster, P. J., Mitchell, J. R., and Karlik, S. J. (2004). In vivo 4.0-t magnetic resonance investigation of spinal cord inflammation, demyelination, and axonal damage in chronic-progressive experimental allergic encephalomyelitis. Journal of Magnetic Resonance Imaging: An Official Journal of the International Society for Magnetic Resonance in Medicine, 20(4):563-571.

Cottaar, M., Wu, W., Tendler, B. C., Nagy, Z., Milller, K., and Jbabdi, S. (2020). Diffusion-prepared phase imaging (dippi): quantifying myelin in crossing fibres. bioRxiv.

Dancause, N., Barbay, S., Frost, S. B., Mahnken, J. D., and Nudo, R. J. (2007). Interhemispheric connections of the ventral premotor cortex in a new world primate. Journal of Comparative Neurology, 505(6):701-715.

Davare, M., Lemon, R., and Olivier, E. (2008). Selective modulation of interactions between ventral premotor cortex and primary motor cortex during precision grasping in humans. The Journal of physiology, 586(11):2735-2742.

Davare, M., Montague, K., Olivier, E., Rothwell, J. C., and Lemon, R. N. (2009). Ventral premotor to primary motor cortical interactions during object-driven grasp in humans. Cortex, 45(9):1050-1057.

Davare, M., Rothwell, J. C., and Lemon, R. N. (2010). Causal connectivity between the human anterior intraparietal area and premotor cortex during grasp. Current Biology, 20(2):176-181.

Doran, M., Hajnal, J. V., Van, N. B., King, M. D., Young, I. R., and Bydder, G. M. (1990). Normal and abnormal white matter tracts shown by mr imaging 
using directional diffusion weighted sequences. Journal of computer assisted tomography, 14(6):865-873.

Dubbioso, R., Madsen, K. H., Thielscher, A., and Siebner, H. R. (2021). The myelin content of the human precentral hand knob reflects inter-individual differences in manual motor control at the physiological and behavioural level. Journal of Neuroscience.

Dum, R. P. and Strick, P. L. (2005). Frontal lobe inputs to the digit representations of the motor areas on the lateral surface of the hemisphere. Journal of Neuroscience, 25(6):1375-1386.

Dutta, D. J., Woo, D. H., Lee, P. R., Pajevic, S., Bukalo, O., Huffman, W. C., Wake, H., Basser, P. J., SheikhBahaei, S., Lazarevic, V., et al. (2018). Regulation of myelin structure and conduction velocity by perinodal astrocytes. Proceedings of the National Academy of Sciences, 115(46):11832-11837.

Elsayed, G. F., Lara, A. H., Kaufman, M. T., Churchland, M. M., and Cunningham, J. P. (2016). Reorganization between preparatory and movement population responses in motor cortex. Nature communications, 7(1):1-15.

Etxeberria, A., Hokanson, K. C., Dao, D. Q., Mayoral, S. R., Mei, F., Redmond, S. A., Ullian, E. M., and Chan, J. R. (2016). Dynamic modulation of myelination in response to visual stimuli alters optic nerve conduction velocity. Journal of Neuroscience, 36(26):6937-6948.

Fischl, B., Salat, D. H., Van Der Kouwe, A. J., Makris, N., Ségonne, F., Quinn, B. T., and Dale, A. M. (2004). Sequence-independent segmentation of magnetic resonance images. Neuroimage, 23:S69-S84.

Fling, B. W., Benson, B. L., and Seidler, R. D. (2013). Transcallosal sensorimotor fiber tract structure-function relationships. Human Brain Mapping, 34(2):384395. 
Forbes, T. A., Goldstein, E. Z., Dupree, J. L., Jablonska, B., Scafidi, J., Adams, K. L., Imamura, Y., Hashimoto-Torii, K., and Gallo, V. (2020). Environmental enrichment ameliorates perinatal brain injury and promotes functional white matter recovery. Nature communications, 11(1):1-17.

Ford, M. C., Alexandrova, O., Cossell, L., Stange-Marten, A., Sinclair, J., KoppScheinpflug, C., Pecka, M., Attwell, D., and Grothe, B. (2015). Tuning of ranvier node and internode properties in myelinated axons to adjust action potential timing. Nature communications, 6(1):1-14.

Forstmann, B. U., Jahfari, S., Scholte, H. S., Wolfensteller, U., van den Wildenberg, W. P., and Ridderinkhof, K. R. (2008). Function and structure of the right inferior frontal cortex predict individual differences in response inhibition: a model-based approach. Journal of Neuroscience, 28(39):97909796.

Friston, K. J., Harrison, L., and Penny, W. (2003). Dynamic causal modelling. Neuroimage, 19(4):1273-1302.

Fünfschilling, U., Supplie, L. M., Mahad, D., Boretius, S., Saab, A. S., Edgar, J., Brinkmann, B. G., Kassmann, C. M., Tzvetanova, I. D., Möbius, W., et al. (2012). Glycolytic oligodendrocytes maintain myelin and long-term axonal integrity. Nature, 485(7399):517.

Glasser, M. F., Coalson, T. S., Robinson, E. C., Hacker, C. D., Harwell, J., Yacoub, E., Ugurbil, K., Andersson, J., Beckmann, C. F., Jenkinson, M., et al. (2016). A multi-modal parcellation of human cerebral cortex. Nature, 536(7615):171-178.

Glasser, M. F., Sotiropoulos, S. N., Wilson, J. A., Coalson, T. S., Fischl, B., Andersson, J. L., Xu, J., Jbabdi, S., Webster, M., Polimeni, J. R., et al. (2013). The minimal preprocessing pipelines for the human connectome project. Neuroimage, 80:105-124. 
Godschalk, M., Lemon, R. N., Kuypers, H. G., and Ronday, H. (1984). Cortical afferents and efferents of monkey postarcuate area: an anatomical and electrophysiological study. Experimental brain research, 56(3):410-424.

Goldman, L. and Albus, J. S. (1968). Computation of impulse conduction in myelinated fibers; theoretical basis of the velocity-diameter relation. Biophysical journal, 8(5):596-607.

Goldsworthy, M., Hordacre, B., and Ridding, M. (2016). Minimum number of trials required for within-and between-session reliability of tms measures of corticospinal excitability. Neuroscience, 320:205-209.

Grandjean, J., Zerbi, V., Balsters, J. H., Wenderoth, N., and Rudin, M. (2017). Structural basis of large-scale functional connectivity in the mouse. Journal of Neuroscience, 37(34):8092-8101.

Hayes, A. F. (2013). Introduction to mediation, moderation, and conditional process analysis: A regression-based approach. 2013. New York: Guilford, 1609182308.

Heath, F., Hurley, S. A., Johansen-Berg, H., and Sampaio-Baptista, C. (2018). Advances in noninvasive myelin imaging. Developmental neurobiology, 78(2):136-151.

Hermundstad, A. M., Bassett, D. S., Brown, K. S., Aminoff, E. M., Clewett, D., Freeman, S., Frithsen, A., Johnson, A., Tipper, C. M., Miller, M. B., et al. (2013). Structural foundations of resting-state and task-based functional connectivity in the human brain. Proceedings of the National Academy of Sciences, 110(15):6169-6174.

Hill, R. A., Li, A. M., and Grutzendler, J. (2018). Lifelong cortical myelin plasticity and age-related degeneration in the live mammalian brain. Nature neuroscience, 21(5):683-695.

Isoda, M. and Hikosaka, O. (2007). Switching from automatic to controlled action by monkey medial frontal cortex. Nature neuroscience, 10(2):240. 
Isoda, M. and Hikosaka, O. (2008). Role for subthalamic nucleus neurons in switching from automatic to controlled eye movement. Journal of Neuroscience, 28(28):7209-7218.

Jenny, A. (1979). Commissural projections of the cortical hand motor area in monkeys. Journal of Comparative Neurology, 188(1):137-145.

Johnen, V. M., Neubert, F.-X., Buch, E. R., Verhagen, L., O'Reilly, J. X., Mars, R. B., and Rushworth, M. F. (2015). Causal manipulation of functional connectivity in a specific neural pathway during behaviour and at rest. Elife, 4:e04585.

Kaller, M. S., Lazari, A., Blanco-Duque, C., Sampaio-Baptista, C., and JohansenBerg, H. (2017). Myelin plasticity and behaviour?connecting the dots. Current opinion in neurobiology, 47:86-92.

Kanai, R. and Rees, G. (2011). The structural basis of inter-individual differences in human behaviour and cognition. Nature Reviews Neuroscience, 12(4):231242.

Kirilina, E., Helbling, S., Morawski, M., Pine, K., Reimann, K., Jankuhn, S., Dinse, J., Deistung, A., Reichenbach, J. R., Trampel, R., et al. (2020). Superficial white matter imaging: Contrast mechanisms and whole-brain in vivo mapping. Science advances, 6(41):eaaz9281.

Kraskov, A., Prabhu, G., Quallo, M. M., Lemon, R. N., and Brochier, T. (2011). Ventral premotor-motor cortex interactions in the macaque monkey during grasp: response of single neurons to intracortical microstimulation. Journal of Neuroscience, 31(24):8812-8821.

Krupnik, R., Yovel, Y., and Assaf, Y. (2021). Inner hemispheric and interhemispheric connectivity balance in the human brain. Journal of Neuroscience, 41(40):8351-8361. 
Kujirai, T., Caramia, M., Rothwell, J. C., Day, B., Thompson, P., Ferbert, A., Wroe, S., Asselman, P., and Marsden, C. D. (1993). Corticocortical inhibition in human motor cortex. The Journal of physiology, 471(1):501-519.

Lakhani, B., Hayward, K. S., and Boyd, L. A. (2017). Hemispheric asymmetry in myelin after stroke is related to motor impairment and function. Neurolmage: Clinical, 14:344-353.

Lang, E. J. and Rosenbluth, J. (2003). Role of myelination in the development of a uniform olivocerebellar conduction time. Journal of neurophysiology, 89(4):2259-2270.

Lanz, F., Moret, V., Ambett, R., Cappe, C., Rouiller, E. M., and Loquet, G. (2017). Distant heterotopic callosal connections to premotor cortex in nonhuman primates. Neuroscience, 344:56-66.

Lazari, A., Giuffre, A., and Nandi, T. (2020a). White matter damage and altered connectivity between primary motor cortices in chronic obstructive pulmonary disease. The Journal of Physiology.

Lazari, A., Koudelka, S., and Sampaio-Baptista, C. (2018). Experiencerelated reductions of myelin and axon diameter in adulthood. Journal of neurophysiology, 120(4):1772-1775.

Lazari, A. and Lipp, I. (2021). Can mri measure myelin? systematic review, qualitative assessment, and meta-analysis of studies validating microstructural imaging with myelin histology. Neurolmage, page 117744.

Lazari, A., Salvan, P., Cottaar, M., Papp, D., van der Werf, O. J., Johnstone, A., Sanders, Z.-B., Sampaio-Baptista, C., Eichert, N., Miyamoto, K., et al. (2020b). Heterogeneous relationships between white matter and behaviour. bioRxiv.

Le, D. B., Turner, R., and Douek, P. (1993). Is water diffusion restricted in human brain white matter? an echo-planar nmr imaging study. Neuroreport, $4(7): 887-890$. 
Lindenberg, R., Nachtigall, L., Meinzer, M., Sieg, M. M., and Flöel, A. (2013). Differential effects of dual and unihemispheric motor cortex stimulation in older adults. Journal of Neuroscience, 33(21):9176-9183.

Lutti, A., Dick, F., Sereno, M. I., and Weiskopf, N. (2014). Using high-resolution quantitative mapping of $\mathrm{r} 1$ as an index of cortical myelination. Neuroimage, 93:176-188.

MacDonald, A. W., Cohen, J. D., Stenger, V. A., and Carter, C. S. (2000). Dissociating the role of the dorsolateral prefrontal and anterior cingulate cortex in cognitive control. Science, 288(5472):1835-1838.

Mancini, M., Karakuzu, A., Cohen-Adad, J., Cercignani, M., Nichols, T. E., and Stikov, N. (2020). An interactive meta-analysis of mri biomarkers of myelin. Elife, 9:e61523.

Mars, R. B., Klein, M. C., Neubert, F.-X., Olivier, E., Buch, E. R., Boorman, E. D., and Rushworth, M. F. (2009). Short-latency influence of medial frontal cortex on primary motor cortex during action selection under conflict. Journal of Neuroscience, 29(21):6926-6931.

Mars, R. B., Piekema, C., Coles, M. G., Hulstijn, W., and Toni, I. (2007). On the programming and reprogramming of actions. Cerebral Cortex, 17(12):29722979.

Matejko, A. A. and Ansari, D. (2015). Drawing connections between white matter and numerical and mathematical cognition: a literature review. Neuroscience \& Biobehavioral Reviews, 48:35-52.

Moore, J. W., Joyner, R. W., Brill, M. H., Waxman, S. D., and Najar-Joa, M. (1978). Simulations of conduction in uniform myelinated fibers. relative sensitivity to changes in nodal and internodal parameters. Biophysical journal, 21(2):147-160. 
Moore, S., Meschkat, M., Ruhwedel, T., Tzvetanova, I. D., Trevisiol, A., Battefeld, A., Kusch, K., Kole, M., Strenzke, N., Möbius, W., et al. (2019). A role of oligodendrocytes in information processing independent of conduction velocity. bioRxiv, page 736975 .

Moseley, M., Cohen, Y., Mintorovitch, J., Chileuitt, L., Shimizu, H., Kucharczyk, J., Wendland, M., and Weinstein, P. (1990a). Early detection of regional cerebral ischemia in cats: comparison of diffusion-and t2-weighted mri and spectroscopy. Magnetic resonance in medicine, 14(2):330-346.

Moseley, M. E., Cohen, Y., Kucharczyk, J., Mintorovitch, J., Asgari, H., Wendland, M., Tsuruda, J., and Norman, D. (1990b). Diffusion-weighted mr imaging of anisotropic water diffusion in cat central nervous system. Radiology, 176(2):439-445.

Movahedian Attar, F., Kirilina, E., Haenelt, D., Pine, K. J., Trampel, R., Edwards, L. J., and Weiskopf, N. (2020). Mapping short association fibers in the early cortical visual processing stream using in vivo diffusion tractography. Cerebral Cortex, 30(8):4496-4514.

Nave, K.-A. (2010). Myelination and support of axonal integrity by glia. Nature, 468(7321):244.

Neubert, F.-X., Mars, R. B., Buch, E. R., Olivier, E., and Rushworth, M. F. (2010). Cortical and subcortical interactions during action reprogramming and their related white matter pathways. Proceedings of the National Academy of Sciences, 107(30):13240-13245.

Oldfield, R. C. et al. (1971). The assessment and analysis of handedness: the edinburgh inventory. Neuropsychologia, 9(1):97-113.

Pajevic, S., Basser, P. J., and Fields, R. D. (2014). Role of myelin plasticity in oscillations and synchrony of neuronal activity. Neuroscience, 276:135-147. 
Papp, D., Callaghan, M. F., Meyer, H., Buckley, C., and Weiskopf, N. (2016). Correction of inter-scan motion artifacts in quantitative $r 1$ mapping by accounting for receive coil sensitivity effects. Magnetic resonance in medicine, 76(5):1478-1485.

Prabhu, G., Shimazu, H., Cerri, G., Brochier, T., Spinks, R. L., Maier, M. A., and Lemon, R. N. (2009). Modulation of primary motor cortex outputs from ventral premotor cortex during visually guided grasp in the macaque monkey. The Journal of physiology, 587(5):1057-1069.

Ridderinkhof, K. R., Ullsperger, M., Crone, E. A., and Nieuwenhuis, S. (2004). The role of the medial frontal cortex in cognitive control. science, 306(5695):443-447.

Romero, M. C., Davare, M., Armendariz, M., and Janssen, P. (2019). Neural effects of transcranial magnetic stimulation at the single-cell level. Nature communications, 10(1):1-11.

Ruckh, J. M., Zhao, J.-W., Shadrach, J. L., van Wijngaarden, P., Rao, T. N., Wagers, A. J., and Franklin, R. J. (2012). Rejuvenation of regeneration in the aging central nervous system. Cell stem cell, 10(1):96-103.

Rushton, W. (1951). A theory of the effects of fibre size in medullated nerve. The Journal of physiology, 115(1):101-122.

Saab, A. S., Tzvetavona, I. D., Trevisiol, A., Baltan, S., Dibaj, P., Kusch, K., Möbius, W., Goetze, B., Jahn, H. M., Huang, W., et al. (2016). Oligodendroglial nmda receptors regulate glucose import and axonal energy metabolism. Neuron, 91(1):119-132.

Sagi, Y., Tavor, I., Hofstetter, S., Tzur-Moryosef, S., Blumenfeld-Katzir, T., and Assaf, Y. (2012). Learning in the fast lane: new insights into neuroplasticity. Neuron, 73(6):1195-1203. 
Salami, M., Itami, C., Tsumoto, T., and Kimura, F. (2003). Change of conduction velocity by regional myelination yields constant latency irrespective of distance between thalamus and cortex. Proceedings of the National Academy of Sciences, 100(10):6174-6179.

Sampaio-Baptista, C., Khrapitchev, A. A., Foxley, S., Schlagheck, T., Scholz, J., Jbabdi, S., DeLuca, G. C., Miller, K. L., Taylor, A., Thomas, N., et al. (2013). Motor skill learning induces changes in white matter microstructure and myelination. Journal of Neuroscience, 33(50):19499-19503.

Sauerbrei, B. A., Guo, J.-Z., Cohen, J. D., Mischiati, M., Guo, W., Kabra, M., Verma, N., Mensh, B., Branson, K., and Hantman, A. W. (2020). Cortical pattern generation during dexterous movement is input-driven. Nature, 577(7790):386-391.

Schauf, C. and Davis, F. A. (1974). Impulse conduction in multiple sclerosis: a theoretical basis for modification by temperature and pharmacological agents. Journal of Neurology, Neurosurgery \& Psychiatry, 37(2):152-161.

Seidl, A. H., Rubel, E. W., and Harris, D. M. (2010). Mechanisms for adjusting interaural time differences to achieve binaural coincidence detection. Journal of Neuroscience, 30(1):70-80.

Sel, A., Verhagen, L., Angerer, K., David, R., Klein-Flügge, M. C., and Rushworth, M. F. (2021). Increasing and decreasing interregional brain coupling increases and decreases oscillatory activity in the human brain. Proceedings of the National Academy of Sciences, 118(37).

Shen, S., Sandoval, J., Swiss, V. A., Li, J., Dupree, J., Franklin, R. J., and Casaccia-Bonnefil, P. (2008). Age-dependent epigenetic control of differentiation inhibitors is critical for remyelination efficiency. Nature neuroscience, 11(9):1024-1034.

Shimazu, H., Maier, M. A., Cerri, G., Kirkwood, P. A., and Lemon, R. N. (2004). Macaque ventral premotor cortex exerts powerful facilitation of motor cortex 
outputs to upper limb motoneurons. Journal of Neuroscience, 24(5):12001211.

Siebner, H. R., Hartwigsen, G., Kassuba, T., and Rothwell, J. C. (2009). How does transcranial magnetic stimulation modify neuronal activity in the brain? implications for studies of cognition. Cortex, 45(9):1035-1042.

Sled, J. G. and Pike, G. B. (2001). Quantitative imaging of magnetization transfer exchange and relaxation properties in vivo using mri. Magnetic Resonance in Medicine: An Official Journal of the International Society for Magnetic Resonance in Medicine, 46(5):923-931.

Smith, R. S. and Koles, Z. J. (1970). Myelinated nerve fibers: computed effect of myelin thickness on conduction velocity. American Journal of PhysiologyLegacy Content, 219(5):1256-1258.

Stagg, C., Bestmann, S., Constantinescu, A., Moreno Moreno, L., Allman, C., Mekle, R., Woolrich, M., Near, J., Johansen-Berg, H., and Rothwell, J. (2011). Relationship between physiological measures of excitability and levels of glutamate and gaba in the human motor cortex. The Journal of physiology, 589(23):5845-5855.

Tabelow, K., Balteau, E., Ashburner, J., Callaghan, M. F., Draganski, B., Helms, G., Kherif, F., Leutritz, T., Lutti, A., Phillips, C., et al. (2019). hmri-a toolbox for quantitative mri in neuroscience and clinical research. Neurolmage.

Tofts, P., Cercignani, M., Tozer, D., Symms, M., Davies, G., Ramani, A., and Barker, G. (2005). Tozer et al. quantitative magnetization transfer mapping of bound protons in multiple sclerosis, magn reson med 2003; 50: 83-91. Magnetic Resonance in Medicine: An Official Journal of the International Society for Magnetic Resonance in Medicine, 53(2):492-493.

Tokuno, H. and Nambu, A. (2000). Organization of nonprimary motor cortical inputs on pyramidal and nonpyramidal tract neurons of primary motor cortex: 
an electrophysiological study in the macaque monkey. Cerebral cortex, 10(1):58-68.

van der Knaap, L. J. and van der Ham, I. J. (2011). How does the corpus callosum mediate interhemispheric transfer? a review. Behavioural brain research, 223(1):211-221.

Verhoeven, K., De Jonghe, P., Van de Putte, T., Nelis, E., Zwijsen, A., Verpoorten, N., De Vriendt, E., Jacobs, A., Van Gerwen, V., Francis, A., et al. (2003). Slowed conduction and thin myelination of peripheral nerves associated with mutant rho guanine-nucleotide exchange factor 10 . The American Journal of Human Genetics, 73(4):926-932.

Wager, T. D., Davidson, M. L., Hughes, B. L., Lindquist, M. A., and Ochsner, K. N. (2008). Prefrontal-subcortical pathways mediating successful emotion regulation. Neuron, 59(6):1037-1050.

Walhovd, K. B., Johansen-Berg, H., and Karadottir, R. T. (2014). Unraveling the secrets of white matter-bridging the gap between cellular, animal and human imaging studies. Neuroscience, 276:2-13.

Waxman, S. G. (1980). Determinants of conduction velocity in myelinated nerve fibers. Muscle \& Nerve: Official Journal of the American Association of Electrodiagnostic Medicine, 3(2):141-150.

Weil, M.-T., Möbius, W., Winkler, A., Ruhwedel, T., Wrzos, C., Romanelli, E., Bennett, J. L., Enz, L., Goebels, N., Nave, K.-A., et al. (2016). Loss of myelin basic protein function triggers myelin breakdown in models of demyelinating diseases. Cell reports, 16(2):314-322.

Weiskopf, N., Edwards, L. J., Helms, G., Mohammadi, S., and Kirilina, E. (2021). Quantitative magnetic resonance imaging of brain anatomy and in vivo histology. Nature Reviews Physics, pages 1-19. 
Weiskopf, N., Suckling, J., Williams, G., Correia, M. M., Inkster, B., Tait, R., Ooi, C., Bullmore, E. T., and Lutti, A. (2013). Quantitative multi-parameter mapping of $\mathrm{r} 1, \mathrm{pd}^{*}, \mathrm{mt}$, and $\mathrm{r}^{*}$ at $3 \mathrm{t}$ : a multi-center validation. Frontiers in neuroscience, 7:95.

Weisskoff, R. M. and Kiihne, S. (1992). Mri susceptometry: image-based measurement of absolute susceptibility of mr contrast agents and human blood. Magnetic resonance in medicine, 24(2):375-383.

Winkler, A. M., Webster, M. A., Brooks, J. C., Tracey, I., Smith, S. M., and Nichols, T. E. (2016). Non-parametric combination and related permutation tests for neuroimaging. Human brain mapping, 37(4):1486-1511.

Yarnykh, V. L. (2002). Pulsed z-spectroscopic imaging of cross-relaxation parameters in tissues for human mri: theory and clinical applications. Magnetic Resonance in Medicine: An Official Journal of the International Society for Magnetic Resonance in Medicine, 47(5):929-939.

Zatorre, R. J., Fields, R. D., and Johansen-Berg, H. (2012). Plasticity in gray and white: neuroimaging changes in brain structure during learning. Nature neuroscience, 15(4):528-536.

Zhao, X., Lynch Jr, J. G., and Chen, Q. (2010). Reconsidering baron and kenny: Myths and truths about mediation analysis. Journal of consumer research, 37(2):197-206.

Zonouzi, M., Scafidi, J., Li, P., McEllin, B., Edwards, J., Dupree, J. L., Harvey, L., Sun, D., Hübner, C. A., Cull-Candy, S. G., et al. (2015). Gabaergic regulation of cerebellar ng2 cell development is altered in perinatal white matter injury. Nature neuroscience, 18(5):674-682. 


\section{Supplementary Files}

This is a list of supplementary files associated with this preprint. Click to download.

- Video1.mov

- NCOMMS2110283BRS.pdf 